\title{
Bulk density of small meteoroids
}

\author{
J.-B. Kikwaya ${ }^{1,2}$, M. Campbell-Brown ${ }^{2}$, and P. G. Brown ${ }^{2}$
}

\author{
1 Vatican Observatory, 00120 Vatican City State, Italy \\ 2 Department of Physics and Astronomy, University of Western Ontario, 1151 Richmond St, London, ON N6A 3K7, Canada \\ e-mail: jbkikwaya@free.fr
}

Received 3 January 2011 / Accepted 22 February 2011

\begin{abstract}
Aims. Here we report on precise metric and photometric observations of 107 optical meteors, which were simultaneously recorded at multiple stations using three different intensified video camera systems. The purpose is to estimate bulk meteoroid density, link small meteoroids to their parent bodies based on dynamical and physical density values expected for different small body populations, to better understand and explain the dynamical evolution of meteoroids after release from their parent bodies.

Methods. The video systems used had image sizes ranging from $640 \times 480$ to $1360 \times 1036$ pixels, with pixel scales from $0.01^{\circ}$ per pixel to $0.05^{\circ}$ per pixel, and limiting meteor magnitudes ranging from $M_{\mathrm{v}}=+2.5$ to +6.0 . We find that $78 \%$ of our sample show noticeable deceleration, allowing more robust constraints to be placed on density estimates. The density of each meteoroid is estimated by simultaneously fitting the observed deceleration and lightcurve using a model based on thermal fragmentation, conservation of energy and momentum. The entire phase space of the model free parameters is explored for each event to find ranges of parameters which fit the observations within the measurement uncertainty.

Results. (a) We have analysed our data by first associating each of our events with one of the five meteoroid classes. The average density of meteoroids whose orbits are asteroidal and chondritic (AC) is $4200 \mathrm{~kg} \mathrm{~m}^{-3}$ suggesting an asteroidal parentage, possibly related to the high-iron content population. Meteoroids with orbits belonging to Jupiter family comets (JFCs) have an average density of $3100 \pm 300 \mathrm{~kg} \mathrm{~m}^{-3}$. This high density is found for all meteoroids with JFC-like orbits and supports the notion that the refractory material reported from the Stardust measurements of 81P/Wild 2 dust is common among the broader JFC population. This high density is also the average bulk density for the 4 meteoroids with orbits belonging to the Ecliptic shower-type class (ES) also related to JFCs. Both categories we suggest are chondritic based on their high bulk density. Meteoroids of HT (Halley type) orbits have a minimum bulk density value of $360_{-100}^{+400} \mathrm{~kg} \mathrm{~m}^{-3}$ and a maximum value of $1510_{-900}^{+400} \mathrm{~kg} \mathrm{~m}^{-3}$. This is consistent with many previous works which suggest bulk cometary meteoroid density is low. SA (Sun-approaching)-type meteoroids show a density spread from $1000 \mathrm{~kg} \mathrm{~m}^{-3}$ to $4000 \mathrm{~kg} \mathrm{~m}^{-3}$, reflecting multiple origins. (b) We found two different meteor showers in our sample: Perseids (10 meteoroids, $\sim 11 \%$ of our sample) with an average bulk density of $620 \mathrm{~kg} \mathrm{~m}^{-3}$ and Northern Iota Aquariids (4 meteoroids) with an average bulk density of $3200 \mathrm{~kg} \mathrm{~m}^{-3}$, consistent with the notion that the NIA derive from $2 \mathrm{P} /$ Encke.
\end{abstract}

Key words. meteorites, meteors, meteoroids - minor planets, asteroids: general - techniques: photometric

\section{Introduction}

Density is one of the most important physical properties of meteoroids. It is a critical factor in modelling the risk meteoroids pose to spacecraft, and also helps determine the physical structure and potentially the chemical composition of meteoroids' parent bodies, comets and asteroids. However, it is difficult to determine meteoroid density from meteor observations, because the meteoroid does not in general ablate uniformly, and does not remain as a single body during its entire flight in the Earth's atmosphere. It experiences fragmentation, which, along with its unknown shape, structure, and chemical composition, complicates any effort of ablation modeling (Kikwaya et al. 2009).

Other factors influence the ablation of a meteoroid, including its entry angle, speed, mass, ablation energy (energy required to ablate a quantity of mass of the meteoroid), boiling temperataure, and bulk density (Campbell-Brown \& Koshny 2004). Each meteoroid will have different ablation behaviour due to its composition, structure, bulk density and size. For example, the beginning heights and lightcurve shapes of faint meteors show significant variation, while larger meteors vary in end height (Ceplecha et al. 1998). Koten et al. (2004) showed that the differences in lightcurves and beginning heights among major video meteor showers can be explained by differences in the chemical composition and physical structure of their parent bodies.

Fragmentation complicates even further the determination of the physical properties of meteoroids, particularly density. The fact that meteoroids fragment is shown by the difference between the meteoroid photometric mass and dynamic mass, observed in the photographic Super-Schmidt meteors, and also in the short trail lengths of Draconid meteors (Jacchia 1955). According to the dustball meteoroid model (Hawkes \& Jones 1975), fragmentation occurs before the process of ablation starts, and the grains undergo intensive ablation after they have been released from the main mass. The dustball model assumes meteoroids are composed of refractory grains held together by a "glue" with a lower boiling point; when this temperature is reached the grains are released. Fragmentation also explains the production of the physical wake in meteor observations. Grains of different 
masses, predicted by the dustball model, decelerate at different rates, leading to an elongated luminous region (Borovicka 2005). Many video meteors observed with short exposures show this wake (Fisher et al. 2000).

The process of fragmentation is complex, and there are several contemporary meteoroid ablation models which try to formalize it. Campbell-Brown \& Koschny (2004) presented an ablation model which follows these steps: (a) the surface temperature of the meteoroid reaches a certain critical point; (b) the binding matrix of the meteroid disrupts (the penetration of the heat through the meteoroid is governed by thermal conductivity); (c) grains are released (fragmentation). Kikwaya et al. (2009) applied this model of ablation to six meteors with masses between $10^{-6}$ and $10^{-8} \mathrm{~kg}$ recorded with narrow-field intensified cameras. They evaluated the bulk density by simultaneously fitting the lightcurve and the deceleration measurements of each of them. They found densities ranging from $500 \mathrm{~kg} \mathrm{~m}^{-3}$ to $7200 \mathrm{~kg} \mathrm{~m}^{-3}$.

Borovicka et al. (2007) used a meteoroid ablation model based on these assumptions: (a) the separation of the meteoroid grains begins after a certain energy flux $\left(10^{6} \mathrm{Jm}^{-2}\right)$ is delivered to the surface of the meteoroid; (b) the meteoroid gradually fragments into grains during the first half of its trajectory; (c) small dynamic pressures fragment the bulk meteoroid not disrupted in the previous step. This ablation model has been successfully applied to six Draconids for which, for the first time, deceleration and photometric measurements were available.

Jacchia (1955) surprisingly found that the dynamical mass of some faint meteoroids decreases more rapidly than the photometric mass as the meteoroid moves along its trajectory, showing unexpected deceleration. To account for this, he introduced the concept of progressive fragmentation (meteoroids fragment progressively into smaller pieces during atmospheric flight). Bellot Rubio et al. (2002) found that visible forms of fragmentation (wake and terminal blending) can be present in the lightcurves of some faint meteors showing deceleration anomalies.

Quantitative measurements of the meteoroid bulk density are difficult, since variations in the way fragmentation is handled in modelling will change the inferred bulk density. Ceplecha (1968) estimated the density of small meteoroids, based on evaluating a parameter $K_{\mathrm{B}}$, which depends on the beginning height of the meteor, the initial velocity and the meteoroid entry-angle. He classified meteoroids into four categories (A-D), with densities ranging from $\mathrm{A}$ (asteroid parent body, $2700 \mathrm{~kg} \mathrm{~m}^{-3}$ ) to $\mathrm{D}$ (cometary parent body, $180 \mathrm{~kg} \mathrm{~m}^{-3}$ ). Babadzhanov (2002) calculated the bulk density of 413 photographic Super-Schmidt using an ablation model based on quasi-continuous fragmentation (QCF). He found values ranging from $400 \mathrm{~kg} \mathrm{~m}^{-3}$ to $7800 \mathrm{~kg} \mathrm{~m}^{-3}$. Bellot Rubio et al. (2002) pointed out two questionable assumptions (see Kikwaya et al. 2009, for details on the discussion) which led Babadzhanov to estimate these high densities, the most important being the fact that Babadzhanov's (2002) method was only based on fitting the lightcurve, neglecting the meteoroid dynamics. Working on the same data, Bellot Rubio et al. (2002), using the single body theory which neglects fragmentation entirely, found bulk densities ranging from $400 \mathrm{~kg} \mathrm{~m}^{-3}$ to $4800 \mathrm{~kg} \mathrm{~m}^{-3}$. The complete model of meteoroid ablation must take into account fragmentation and dynamical properties of the meteoroids simultaneously (Borovicka 2005), as described and applied by Borovicka et al. (2007), and Kikwaya et al. (2009).

From these previous works, we see that estimating the density of a meteoroid is a difficult task, but it is achievable by combining precise observations (both photometric and astrometric measurements) and a complete model of ablation where all the different processes of mass-loss are taken into account (Hill et al. 2005; Popova 2004; Borovička et al. 2007; Jones \& Kaiser 1966). Strictly speaking, this type of analysis should produce a bulk density for meteoroids not as a single and unique value, but instead as a range of possible solutions.

The closest direct analog to small meteoroids to have directly measured densities are IDPs. Love et al. (1994) measured the densitiess of about 150 5-15 $\mu \mathrm{m}$ sized stratospheric IDPs (Interplanetary Dust Particles). Masses were determined using an absolute X-ray analysis technique with a transmission electron microscope, and volumes were found using scanning electron microscope imagery. They found that unmelted chondritic particles have densities between $500 \mathrm{~kg} \mathrm{~m}^{-3}$ and $6000 \mathrm{~kg} \mathrm{~m}^{-3}$. Almost half of the particles had densities below $2000 \mathrm{~kg} \mathrm{~m}^{-3}$, and porosity which is significant, but less than 70\%. IDPs with densities above $3500 \mathrm{~kg} \mathrm{~m}^{-3}$ contain large sulfide grains. Melted chondritic particles (chondritic spherules) were found to have densities near $3500 \mathrm{~kg} \mathrm{~m}^{-3}$. They find a peak in IDP density for debiased exoatmospheric population near $2000 \mathrm{~kg} \mathrm{~m}^{-3}$, with significant numbers up to $\sim 7000 \mathrm{~kg} \mathrm{~m}^{-3}$ (see Fig. 5 of Love et al. 1994).

In this work, following Kikwaya et al. (2009), we use the dustball theory as modelled numerically by Campbell-Brown \& Koshny (2004), and we apply it to faint (magnitude +2.5 to +6.0 ) meteors. We will explore the entire solution space of each of 8 free model parameters, and we will produce for each meteor typically hundreds of thousands of solutions. Each solution consists of a lightcurve and deceleration curve (variation of velocity with height) which will be compared to the observed lightcurve and deceleration. We use the reduced $\chi_{\mathrm{c}}^{2}$ value of the observed versus modeled astrometry and photometry as figure of merit (for a detailed discussion of the use and application of the reduced $\chi_{\mathrm{c}}^{2}$ value, see Bevington \& Robinson 2003).

\section{Observations and equipment}

Data were collected with two or three stations in seven different runs between 2006 and 2009: one over six nights with two stations in Arizona, USA, in 2006; five runs with two stations over fourteen nights in Canada from 2007 to 2009; and one run with three stations over two nights in Canada in 2009. Three different systems were used: third generation (Gen III) intensified cameras, a digital Image Intensified CCD camera with an electronically gated image intensifier (referred to hereafter as the gated system), and a high resolution Gen III system, hereafter Cooke camera.

The Gen III cameras use ITT NiteCam model 380i microchannel plate image intensifiers with $18 \mathrm{~mm}$ GaAs detectors. These are lens coupled to Cohu 4910 series video CCD cameras, which are high performance, relatively sensitive, monochrome CCD cameras with analogue output (Hawkes et al. 2001; Hawkes 2002), having $480 \times 640$ pixels recording at 30 interlaced frames per second. We used two of these systems, each with a $25-\mathrm{mm}$ Fujinon f/0.85 lens as the objective. A single gated system with integrated intensifier was used, with a $50-\mathrm{mm}$ Fujinon $\mathrm{f} / 0.75$ objective lens. The gated camera CCD had a pixel array of $1360 \times 1036$ pixels and 14 bit photometric depth. An internal electronic device, set by an external control box, controls both the CCD exposure time and the rate of the gating. The gating rate is much higher than the frame rate, so a meteor is broken into a series of dots (see Fig. 1). During all seven campaigns which used the gated system, the CCD exposure time 
Table 1. Summarizing all campaigns and their details.

\begin{tabular}{|c|c|c|c|c|c|c|c|c|}
\hline Camp. Duration & \# Usable nights & \# Stations & & Location & & Baseline & Instruments & $\mathrm{Nb}$ events \\
\hline \multirow{4}{*}{ 20060430-20060506 } & \multirow{4}{*}{5} & \multirow{4}{*}{2} & site 1 & site 2 & site 3 & \multirow{4}{*}{$77.04 \mathrm{~km}$} & & \multirow{4}{*}{09} \\
\hline & & & Kitt Peak & Whipple obs & & & Gen III camera & \\
\hline & & & $31^{\circ} 57^{\prime} 70 \mathrm{~N}$ & $31^{\circ} 40^{\prime} 51 \mathrm{~N}$ & & & Gated system & \\
\hline & & & $111^{\circ} 36^{\prime} 01 \mathrm{~W}$ & $110^{\circ} 57^{\prime} 17 \mathrm{~W}$ & 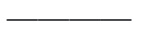 & & Gen III camera & \\
\hline \multirow{3}{*}{$20070420-20070422$} & \multirow{3}{*}{2} & \multirow{3}{*}{2} & Elginfied & Tavistock & & \multirow{3}{*}{$44.61 \mathrm{~km}$} & Gen III camera & \multirow{3}{*}{05} \\
\hline & & & $43^{\circ} 11^{\prime} 58 \mathrm{~N}$ & $43^{\circ} 15^{\prime} 84 \mathrm{~N}$ & - & & Gated system & \\
\hline & & & $81^{\circ} 18^{\prime} 90 \mathrm{~W}$ & $80^{\circ} 46^{\prime} 32 \mathrm{~W}$ & L & & Gen III camera & \\
\hline \multirow{3}{*}{ 20070519-20070520 } & \multirow{3}{*}{1} & \multirow{3}{*}{2} & Elginfied & Tavistock & & \multirow{3}{*}{$44.61 \mathrm{~km}$} & Gen III camera & \multirow{3}{*}{05} \\
\hline & & & $43^{\circ} 11^{\prime} 58 \mathrm{~N}$ & $43^{\circ} 15^{\prime} 84 \mathrm{~N}$ & & & Gated system & \\
\hline & & & $81^{\circ} 18^{\prime} 90 \mathrm{~W}$ & $80^{\circ} 46^{\prime} 32 \mathrm{~W}$ & & & Gen III camera & \\
\hline \multirow{3}{*}{$20070812-20070813$} & \multirow{3}{*}{2} & \multirow{3}{*}{2} & Elginfied & Tavistock & & \multirow{3}{*}{$44.61 \mathrm{~km}$} & Gen III camera & \multirow{3}{*}{17} \\
\hline & & & $43^{\circ} 11^{\prime} 58 \mathrm{~N}$ & $43^{\circ} 15^{\prime} 84 \mathrm{~N}$ & & & Gated system & \\
\hline & & & $81^{\circ} 18^{\prime} 90 \mathrm{~W}$ & $80^{\circ} 46^{\prime} 32 \mathrm{~W}$ & & & Gen III camera & \\
\hline \multirow{3}{*}{ 20080910-20080911 } & \multirow{3}{*}{2} & \multirow{3}{*}{2} & Elginfied & Tavistock & & \multirow{3}{*}{$44.61 \mathrm{~km}$} & Gen III camera & \multirow{3}{*}{25} \\
\hline & & & $43^{\circ} 11^{\prime} 58 \mathrm{~N}$ & $43^{\circ} 15^{\prime} 84 \mathrm{~N}$ & & & Gated system & \\
\hline & & & $81^{\circ} 18^{\prime} 90 \mathrm{~W}$ & $80^{\circ} 46^{\prime} 32 \mathrm{~W}$ & & & Gen III camera & \\
\hline \multirow{3}{*}{$20090625-20090911$} & \multirow{3}{*}{8} & \multirow{3}{*}{2} & Elginfied & Tavistock & & \multirow{3}{*}{$44.61 \mathrm{~km}$} & Cooke camera & \multirow{3}{*}{31} \\
\hline & & & $43^{\circ} 11^{\prime} 58 \mathrm{~N}$ & $43^{\circ} 15^{\prime} 84 \mathrm{~N}$ & & & Gated system & \\
\hline & & & $81^{\circ} 18^{\prime} 90 \mathrm{~W}$ & $80^{\circ} 46^{\prime} 32 \mathrm{~W}$ & & & Cooke camera & \\
\hline \multirow{3}{*}{ 20090624-20090825 } & \multirow{3}{*}{3} & \multirow{3}{*}{3} & Elginfied & Tavistock & Brussels & $61.36 \mathrm{~km}$ & Cooke camera & \\
\hline & & & $43^{\circ} 11^{\prime} 58 \mathrm{~N}$ & $43^{\circ} 15^{\prime} 84 \mathrm{~N}$ & $43^{\circ} 44^{\prime} 88 \mathrm{~N}$ & $44.61 \mathrm{~km}$ & Gated system & 15 \\
\hline & & & $81^{\circ} 18^{\prime} 90 \mathrm{~W}$ & $80^{\circ} 46^{\prime} 32 \mathrm{~W}$ & $81^{\circ} 12^{\prime} 42 \mathrm{~W}$ & $64.14 \mathrm{~km}$ & Cooke camera & \\
\hline
\end{tabular}

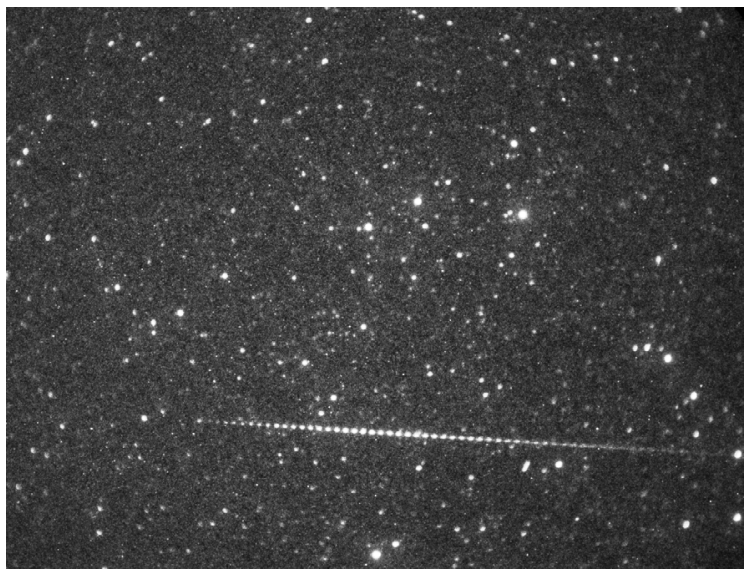

Fig. 1. Meteor 20090825-065903 recorded with the gated camera on August 25, 2009 at 06:59:03 UTC at Brussels in Ontario in Canada. The frequency of CCD was set to $5 \mathrm{~Hz}$ and the frequency of the intensifier gating was $100 \mathrm{~Hz}$.

was set to 5 frames per second ( $5 \mathrm{~Hz}$ ). From 2006 to 2007, we used $200 \mathrm{~Hz}$ for the gating, and from 2008 to 2009, to ensure that dots were well separated, we used $100 \mathrm{~Hz}$. The high resolution digital intensified CCD cameras, our third system, used the same Gen III ITT intensifiers as the Gen III system lens coupled to a Cooke pco.1600 CCD camera, which are run with a resolution of $1024 \times 1024$ pixels at $20 \mathrm{fps}$ (progressive scan). These cameras have digital output and 14-bit optical depth. Two of these were used, each with $50 \mathrm{~mm}$ f/0.95 Navitar objective lens (see Tables 1 and 2).

\section{Data and reduction}

We obtained a total of 107 useful multistation meteors over all seven campaigns from 2006 to 2009 . By useful, we mean that the triangulation geometry was good (large angular separation of observing planes from the two stations), and the SNR was high enough to make precise measurements. After each campaign, we searched the data for simultaneous meteors, and checked if the meteor started and ended in the field of view of at least one of the stations: all partial trails were rejected. When the gated camera was used, we also required the meteor to be captured by the gated camera to provide precise astrometric measurements. For photometric measurements, we used only two systems: either the Gen III or high-resolution Cooke systems. The gated camera was not used for photometry because the meteor images in each frame are so close together that their photometric measurements blend into nearby meteor "dots".

For a better estimate of the photometric mass of the meteor, we added to the astrometric critera the requirement that the event should start in the field of view of at least one Gen III or highresolution system, as this gives a complete lightcurve of the meteor. After applying these criteria, the total number of useful meteors went down to 92 .

\subsection{Astrometry}

We used standard astrometric techniques (Kikwaya et al. 2009) to extract the positions of meteors by comparing them to the positions of stars. For the Gen III systems, typical errors of $0.016^{\circ}$ are found by comparing plate fits with actual positions, which corresponds, at the Gen III resolution, to $\sim 0.3$ pixels. On the gated system, the plate errors were approximately $0.007^{\circ}$ (0.6 pixels) and on the Cooke camera system, errors were around $0.012^{\circ}$, or approximately 0.3 pixels.

The astrometric procedures used in Kikwaya et al. (2009) were also used in this study. Briefly, the meteor positions were measured manually and the velocities smoothed by choosing frames 5 to 25 points apart, depending on the system, to clearly show the deceleration. The atmospheric trajectories were calculated using the computer program Milig, based on the algorithm described in Borovicka (1990).

As an example, we compute the trajectory solution with Milig for meteor 20060503-091349, recorded on May 3, 2006 


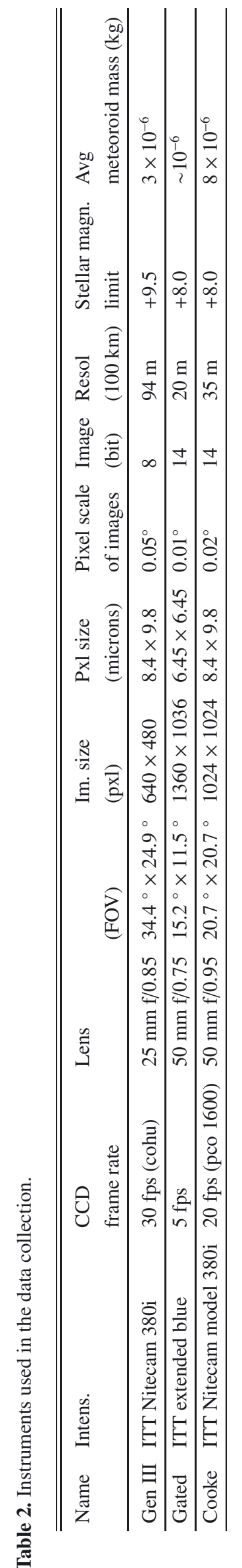

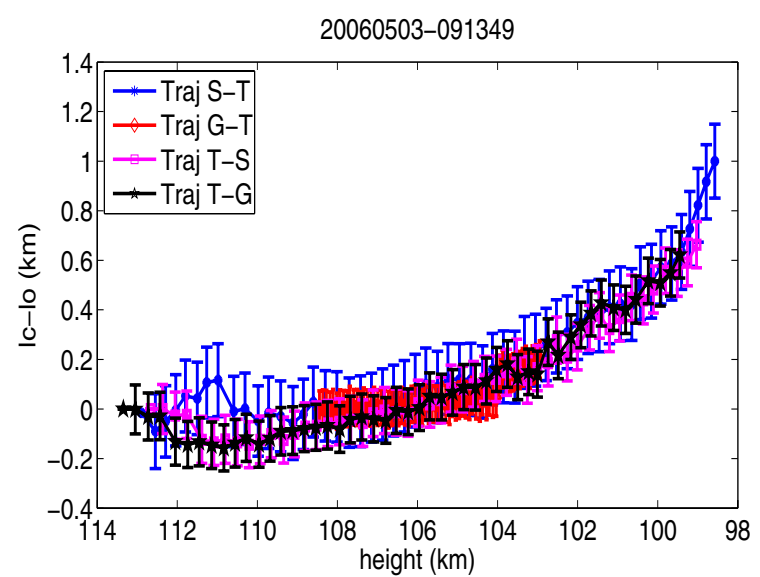

Fig. 2. Plot showing the deceleration of the meteor 20060503-091349. Here $l_{\mathrm{o}}$ is the observed length of the meteor along its trajectory and $l_{\mathrm{c}}$ is the calculated length assuming a velocity of $v=61 \mathrm{~km} \mathrm{~s}^{-1}\left(v=\frac{l_{c}}{t}, t\right.$ being the meteor time). Traj S-T is the trajectory solution from camera $\mathrm{S}$ computed using the cameras $\mathrm{S}$ and T; Traj T-S is the trajectory solution from camera $\mathrm{T}$ computed using the cameras $\mathrm{S}$ and $\mathrm{T}$. Traj G-T is the trajectory solution from camera $\mathrm{G}$ computed using the cameras $\mathrm{G}$ and $\mathrm{T}$ and Traj T-G is the trajectory solution from camera $\mathrm{T}$ computed using the camera $\mathrm{G}$ and $\mathrm{T}$.

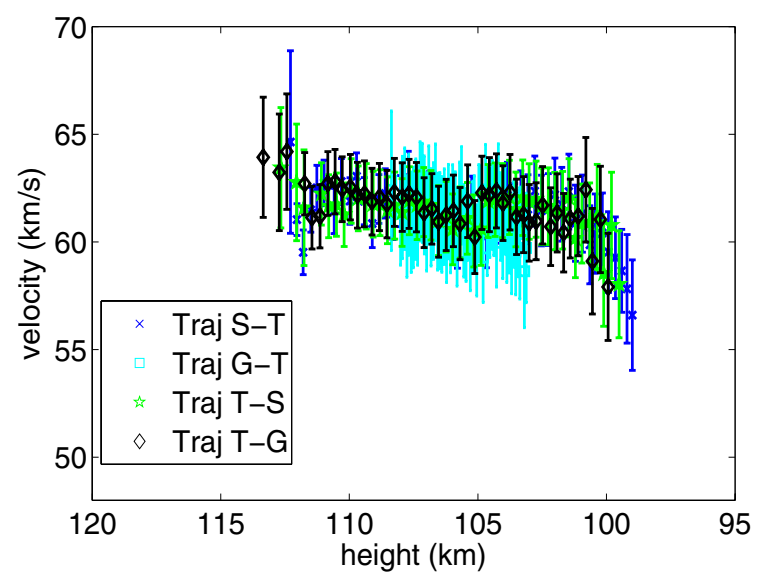

Fig. 3. Velocity profile of the meteor 20060503-091349. We have inserted errorbars at each point along the trajectory. The first points are simple point-to-point differencing while the remaining points are several points smoothed: 5 for Gen III and 10 for gated camera (see Fig. 2 for legend).

at 09:13:49 UTC in Tucson, Arizona. The deceleration, necessary to constrain the model, was noticeable. The deceleration is more obvious when we calculate the difference between the computed distance (assuming a constant speed) and the observed distance (Fig. 2). The smoothed curve of the velocity of the meteor 20060503-091349 is shown in Fig. 3. The uncertainty at each point has been calculated for later use in comparing with the model data.

The pre-atmospheric velocity was determined by taking a linear least-squares fit of the first 5 to 25 positions (equal to the number of points used in smoothing the curve). The error on the pre-atmospheric velocity is the standard deviation of the initial velocity on the two or three cameras which recorded it. For meteor 20060503-091349, the pre-atmospheric speed was $61.30 \pm 0.99 \mathrm{~km} \mathrm{~s}^{-1}$.

The uncertainties for each velocity measurement (Fig. 3) come from the combination of systematic and random error of the position picks for the meteor in each frame. We found the 
systematic error by comparing the positions of 10 meteors, reduced separately by 9 people. Each person introduces a different systematic shift, which we found to be 0.6 pixels on average; this was adopted as the systematic error for all the systems. The random error is evaluated from the observed standard deviation of the points (residuals) for a two station solution, since this represents the scatter about a line. The random error depended on the system: for the Gen III cameras, the random error ranged from 0.2 to 0.3 pixels; for the gated camera, it was between 0.4 and 0.6 pixels; and for the Cooke camera, the random error was about 0.5 pixels. We added these two types of error to all measured points of each meteor in a Monte Carlo simulation, which was run between 2500 to 5000 times (each point had a gaussian error distributed according to these values). We were then able to determine the uncertainty in position, height, range and velocity for each meteor taking the camera-meteor-camera geometry explicitly into account.

The heliocentric meteoroid orbits were then computed using the methods of Ceplecha (Ceplecha 1987).

\subsection{Photometry}

We used standard routines for meteor photometry, comparing the integrated brightness above background of the stars in a flat-fielded image to the brightness of each meteor image (cf. Kikwaya et al. 2009). A total of about 100 stars are used in each calibration; the $V$ magnitudes are taken from the SKY2000v4 catalog (Myers et al. 2001). Although the Gen III and the Cooke systems have a spectral response closer to the $R$ band than the $V$ band (around 45\% quantum efficiency in $R$ compared to $35 \%$ in $V$; also the stellar $R$ magnitudes give a calibration fit with less scatter than the stellar $V$ magnitudes), we use the stellar $V$ magnitudes to calibrate. The luminous efficiency (cf. Hill et al. 2005) used to compute the photometric mass of meteors has been estimated in the panchromatic band, and not the red (Ceplecha et al. 2000; Koten et al. 2006). To check for errors introduced when using $V$ magnitudes instead of $R$, we took a sample of meteor spectra from Borovicka et al. (2005), and worked out an average correction factor to convert the $R$-band meteor magnitude to a $V$-band meteor magnitude. This average $(R-V)$ value was $0.72 \mathrm{mag}$. We calculated light curves in the $R$-band for a few of our meteors, and used the correction factor to convert these to $V$ magnitudes. We found that the lightcurves were the same within our error as those found using the $V$-band calibration directly, so we used the $V$-band calibration for all our meteors.

A typical error of $0.1-0.3 \mathrm{mag}$ is found for each star. It represents the difference between the catalogue value and the calibration. At fainter magnitudes, this error is dominated by counting statistics and shot noise in the intensifier. At brighter magnitudes, the error is mainly due to uncertainty in the photometric calibration. The combination of Poisson error and error in the calibration fit are used to estimate the calibration uncertainty in the meteor magnitudes (cf. Kikwaya et al. 2010). The lightcurve of each meteor is calculated as apparent magnitude, and converted to absolute magnitude, defined as the apparent brightness the meteor would have at a range of $100 \mathrm{~km}$ at the zenith.

The Gen III systems, with 8-bit pixel depth, were subject to saturation effects for meteors +2.3 mag and brighter. The maximum pixel value in this case is truncated to 255 , and blooming occurs as the excess photoelectrons spill over into neighbouring pixels. This saturation effect flattens the lightcurves of bright meteors, which has serious consequences for attempts to

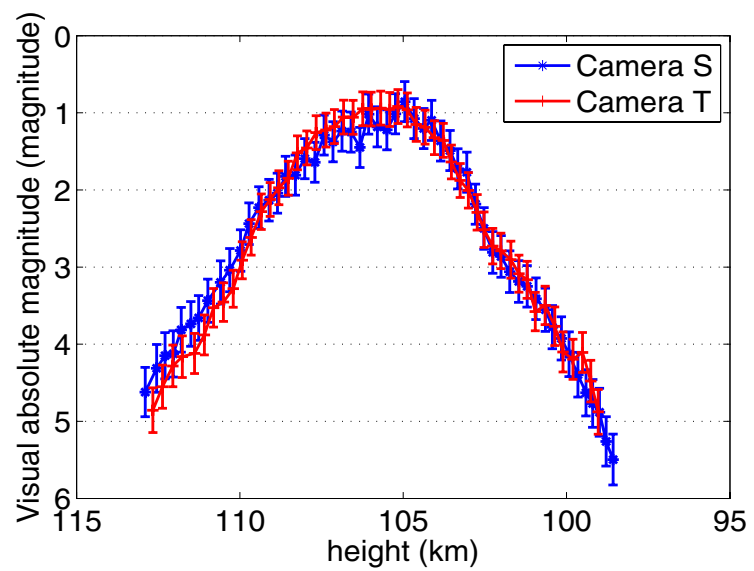

Fig. 4. The absolute lightcurves of the meteor 20060503-091349 obtained after applying the different photometric techniques (the correction for range has been applied).

fit the lightcurve. For the nine meteors in our sample which were saturated through part of their lightcurves, a saturation correction (Kikwaya et al. 2010) was applied to extract the true magnitude for those frames. The typical maximum correction to a lightcurve was approximatly $+1 \mathrm{mag}$, a significant effect. Saturation was not a factor in the Cooke system, because of the much larger bit depth.

Figure 4 shows the lightcurve of the meteor 20060503091349 from two Gen III cameras.

\section{Ablation model}

We use the dustball model of Campbell-Brown \& Koschny (2004). It uses the Clausius-Clapeyron formalism to consider mass loss before the boiling temperature is reached, and allows the user to define the number and size of constituent grains. The model considers three sources of energy:

1. energy imparted to the meteoroid by collisions with atmospheric atoms;

2. energy lost from the meteoroid by radiation;

3. energy lost through evaporation of the meteoroid material.

The equation of the momentum transfer from air molecules which collide with the meteoroid is given by:

$\frac{\mathrm{d} v}{\mathrm{~d} t}=\frac{\Gamma \rho_{\mathrm{a}} v^{2}}{m} A\left(\frac{m}{\rho_{\mathrm{m}}}\right)^{2 / 3}$

where $\rho_{\mathrm{a}}$ is the atmospheric density, $\rho_{\mathrm{m}}$ is the density of the meteoroid, and $A$ is the shape factor of the meteoroid, defined so that $A\left(m / \rho_{\mathrm{m}}^{2 / 3}\right)$ is the effective cross sectional area of the meteoroid. $\Gamma$ is a dimensionless coefficient, varying between 0 and 1 and describing the proportion of momentum from air molecules transfered to the meteoroid. The velocity of the meteoroid, output versus both time and height, provides the deceleration.

The classical meteor ablation model considers mass loss to be proportional to the kinetic energy imparted to the meteoroid. The ablation in our model starts as soon as the surface of the meteoroid reaches the boiling temperature (e.g. Bronshten 1983), which means as the classical model of ablation suggests, when the "glue" evaporates and grains are released. The classical equation of mass loss is then given by:

$\frac{\mathrm{d} m}{\mathrm{~d} t}=-\frac{A \Lambda}{2 L}\left(\frac{m}{\rho_{\mathrm{m}}}\right)^{2 / 3} \rho_{\mathrm{a}} v^{3}$ 
where $\Lambda$, the heat-transfer coefficient, is equal to or less than unity, since the energy expended on ablation cannot exceed the total kinetic energy of the oncoming stream molecules (Bronshten 1983), and $L$ is the heat of ablation (energy that must be delivered to a mass $\mathrm{d} m$ in order to melt and/or vaporize it).

The ablation model we use in our work considers ablation as soon as the meteoroid begins to heat, very high in the atmosphere. The mass loss is then calculated through KnudsenLangmuir formula combined with the Clausius-Clapeyron equation, adding a term like the classical formula to simulate spallation when the meteoroid is very hot. The mass loss equation is evaluated as:

$$
\frac{\mathrm{d} m}{\mathrm{~d} t}=A\left(\frac{m}{\rho_{\mathrm{m}}}\right)^{2 / 3} \psi \frac{P_{\mathrm{a}} \exp \left(\frac{L \mu}{k_{\mathrm{B}} T_{\mathrm{M}}}\right) \exp \left(-\frac{L \mu}{k_{\mathrm{B}} T_{\mathrm{B}}}\right)-p_{\mathrm{v}}}{\sqrt{\frac{2 \pi k_{\mathrm{B}} T}{\mu}}}
$$

where $p_{\mathrm{v}}$ is the vapor produced by sublimating meteoroid material and $\psi$ is the condensation coefficient, which gives the probability that an ablated meteor atom colliding with the surface will condense. For metals, $\psi$ is 1: following Bronshten (1983), $\psi$ of stone is considered to be $0.5 . T_{\mathrm{B}}$ is the boiling temperature of the meteoroid substance at sea level pressure $P_{\mathrm{a}}$, and $T_{\mathrm{M}}$ is the meteoroid temperature (we note a typographic error in the Eq. (9) of Campbell-Brown \& Koschny 2004, where $T_{\mathrm{B}}$ in the first expression term should be $T_{\mathrm{M}}$ ). $L$ is the heat of ablation, $\mu$ the average mass of a meteor atom and $k_{\mathrm{B}}$ the Boltzmann constant. For more details of this particular equation of mass loss and the physical background behind it application, see Campbell-Brown \& Kosheny (2004).

\section{Analysis}

For each of our 92 meteors, we extracted the trajectory and light curve, and applied our ablation model to simultaneously fit the observed lightcurve and deceleration.

Among the parameters used in the model, we have chosen some to remain fixed and some to remain free. Fixed parameters include:

1. the fusion point of the meteoroid grains (we constrain it to be $100 \mathrm{~K}$ less than the boiling point of the meteoroid grains);

2 . the condensation coefficient $\psi$ (fixed to 0.5: condensation coefficient of stone) (Campbell-Brown \& Koschny 2004);

3. the emissivity of the meteoroid (fixed to 0.9) (Campbell-Brown \& Koschny 2004);

4. the atmospheric density, provided by the MSISE-90 model (Hedin 1991).

For each meteor, we search the entire free parameter space in two stages. The first, broad search identifies the regions in which solutions are concentrated. For this first step, we use a relaxed fit criterion requiring agreement between the model and two thirds of common points in the lightcurve and four fifths of the measured points in the deceleration, since the grain distribution is not varied at this step and the shape of the lightcurve may be a poor fit. If all of the good solutions have densities which are grouped within $\pm 1000 \mathrm{~kg} / \mathrm{m}^{-3}$ (typical of the range of density errors reported in other works, such as Babadzhanov 2002, see Table 1; Bellot Rubio et al. 2002, see Table 2; and Kikwaya et al. 2009, see Table 10) we take the best solution among them. Our figure of merit for defining the best model fit is the model with the reduced chi-squared which is closest to unity. If we find good solutions with a wider range in densities, we choose the best solution in each group of similar density and attempt to find a "best" solution within each group. The reason for beginning with this coarse search is the difficulty of determining the grain distribution; if the number of solutions covering a reasonable range of each of the other free parameters is merely prohibitive, the number including all possible grain distributions is essentially infinite.

In the broad search, the approximate grain mass distribution index is estimated using the work of Murray $\&$ Beech as a guide: they associated the shape of a lightcurve (characterized by the F-parameter) to a meteoroid mass distribution index (Murray \& Beech 2000; see Table 2). The grain mass distribution may not follow the true mass index of each of our meteoroids, but it provides a starting point for the broad search of parameter space. Murray \& Beech (2000) assume the grain mass index is selfsimilar to the meteoroid population mass distribution as a whole, i.e. that the grain mass index is the same as the meteor mass index, s. With this mass index, grains are distributed into three bins according to the method described by Borovička et al. (2007). Only three bins are used to minimize computing time, which is already very large.

Once the best "coarse" solution is found in the broad search, the grain masses are forward modelled to give the best fit to the shape of the lightcurve and the deceleration. If several best densities were found, this procedure was repeated for each group of densities; in each case, it happened that we could rule out all but one of the density groups at this stage, since no good match could be found for the others. This grain distribution was then taken to be the final distribution for a particular meteoroid. At this point, we have a best fit "coarse" density, but in order to evaluate the error in this density measure, we will once again search the entire free parameter space with a smaller range in density and higher resolution in density (refined search). All solutions from the refined search are sorted by reduced chi-squared using both the lightcurve and deceleration simultaneously, and those with reduced chi-squared of one or less we adopt as the density distribution of the meteoroid.

\subsection{Broad search}

\subsubsection{Free parameters}

In our first stage, the broad search, the free parameters of our ablation model include:

- grain mass and number of grains;

- density of the meteoroid;

- heat of ablation;

- boiling point of the meteoroid grains $\left(T_{\text {boil }}\right)$;

- temperature at which the grains are released $\left(T_{\mathrm{lim}}\right)$;

- specific heat $\left(C_{\mathrm{p}}\right)$;

- average molar mass of meteoroid material;

- thermal conductivity of the meteoroid.

The range of values for each free parameter is described in Table 3.

In most models, $\Gamma$ (drag coefficient) and $\Lambda$ (heat transfer coefficient) are assumed. Borovicka et al. (2007) set both parameters to unity; this was also the value assumed by Fisher et al. (2000). Campbell-Brown \& Koschny (2004) assumed $\Gamma=1$ and $\Lambda=0.5$. We treat $\Gamma$ and $\Lambda$ differently during the broad search and at the refined one. In the broad search, they are set to unity. When fitting the grain distribution, we manually adjust $\Gamma$ and $\Lambda$ to improve the model fit. For the refined search, we allow $\Gamma$ and $\Lambda$ to vary from 0.5 to 1.0 . 
Table 3. Free parameters of the model and the range of values used.

\begin{tabular}{cccc}
\hline \hline Parameter & Range & Step & References \\
\hline $\begin{array}{c}\text { Density } \\
\left(\mathrm{kg} \mathrm{m}^{-3}\right)\end{array}$ & $100-8000$ & 300 & $\begin{array}{c}\text { very porous Cabornaceous- } \\
\text { iron meteoroid }\end{array}$ \\
\hline $\begin{array}{c}\text { Heat of Ablation } \\
\left(\mathrm{J} \mathrm{kg}^{-1}\right)\end{array}$ & $2 \times 10^{6}-9 \times 10^{6}$ & $1 \times 10^{6}$ & $\begin{array}{c}\text { Hawkes \& Jones (1975) } \\
\text { Bellot Rubio et al. (2002) } \\
\text { Borovicka et al. (2007) } \\
\text { Kikwaya et al. (2009) }\end{array}$ \\
\hline $\begin{array}{c}\text { Fragmentation T } \\
(\mathrm{K})\end{array}$ & $900-1600$ & 100 & $\begin{array}{c}\text { Campbell-Brown \& Koschny (2004) } \\
\text { Kikwaya et al. (2009) }\end{array}$ \\
\hline Boiling T (K) & $1400-2300$ & 200 & Campbell-Brown \& Koschny (2004) \\
\hline $\begin{array}{c}\text { Thermal conductivity } \\
\left(\mathrm{W} \mathrm{m} \mathrm{m}^{-1} \mathrm{~K}^{-1}\right)\end{array}$ & $0.1-1$ & 0.1 & $\begin{array}{c}\text { Campbell-Brown \& Koschny (2004) } \\
\text { Popova (2005) }\end{array}$ \\
& & & $\begin{array}{c}\text { Kikwaya et al. (2009) } \\
\text { Vokrouhlicky \& Farinella (2000) }\end{array}$ \\
\hline Molar mass (amu) & $20,36,56$ & & Sodium - iron \\
\hline Specific heat $\left(\mathrm{J} \mathrm{kg}^{-1} \mathrm{~K}^{-1}\right)$ & $600-1400$ & 200 & Campbell-Brown \& Koschny (2004) \\
\hline
\end{tabular}

\subsubsection{Grain mass distribution}

For the initial broad search, we used three grain masses for each meteor. The smallest mass was close to the ablation limit, below which radiation will dominate and the mass loss will be negligible. The other masses, and the number of grains in each bin, were assigned according to the mass distribution index using the method of Borvicka et al. (2007). The total mass was made to agree with the photometric mass determined for each meteor. To determine photometric mass, we used the luminous efficiency as described by Hill et al. (2005).

To determine a trial mass index for each meteor, we used the work of Murray \& Beech (2000), who related the F-parameter (a standard measure of the asymmetry of a light curve) to the mass index of grains which could successfully fit the curve. Although this was often not the final grain distribution, nor a unique solution, it did provide a useful starting point.

The F-parameter is defined as the ratio of the distance from the beginning to the point of maximum brightness to the full length of the light curve: an F-parameter smaller than 0.5 indicates the light curve peaks early, greater than 0.5 indicates that it peaks late, and 0.5 represents a symmetrical lightcurve. For consistency, the F-parameter is generally measured some fixed number of magnitudes below the peak rather than from the true beginning and ending of the curve; we used the F-parameter measured from 0.75 mag below the peak. This value was chosen because it allowed the parameter to be calculated even for faint meteors. Murray \& Beech (1999) reported values measured from three different magnitudes below the peak: 0.5, 0.75 and 1.0.

The grain mass indices of our meteors were found to range from 1.50 to 2.85 using this approach. The smallest grains were typically $10^{-12} \mathrm{~kg}$, and the largest grains used were $10^{-9} \mathrm{~kg}$.

Using the grain distribution above and the range of values for each free parameter previously described, we computed about a hundred thousand models for each meteor, and compared the model lightcurves and deceleration to the measured curves (Figs. 4 and 3). We defined a good fit as one where the reduced chi-squared between the model values for the lightcurve and velocity as function of height as compared to the observations were less than or equal to unity. We accepted at this stage an overlap of at least two thirds of points between the modelled values and the observed ones. Figure 5 shows an example (meteor 20060503091349) of the density distribution of all solutions with $\chi_{\mathrm{c}}^{2}<1$;

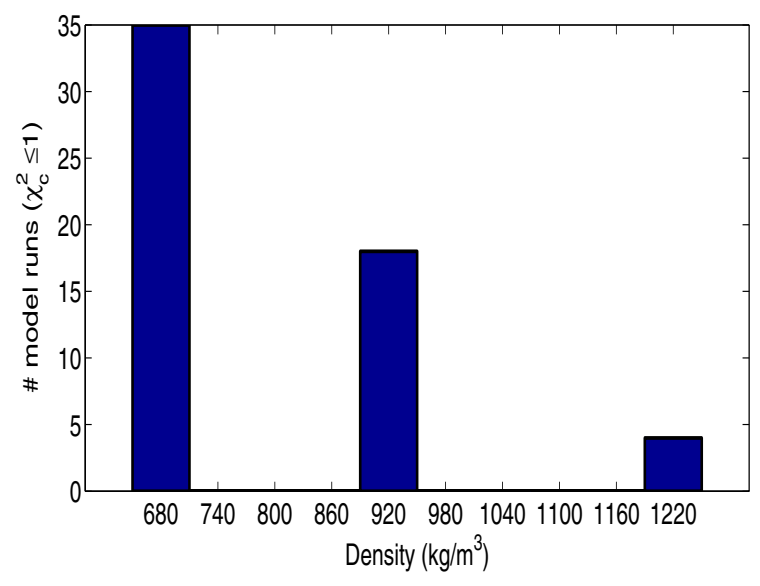

Fig. 5. All model solutions with $\chi_{\mathrm{c}}^{2} \leq 1$ after the initial broad search for meteor 20060503-091349.

there are solutions around densities of $680 \mathrm{~kg} \mathrm{~m}^{-3}, 920 \mathrm{~kg} \mathrm{~m}^{-3}$ and $1220 \mathrm{~kg} \mathrm{~m}^{-3}$.

If the best fits were closely grouped, we chose the one having the $\chi_{\mathrm{c}}^{2}$ closest to 1 for both the lightcurve and deceleration for inclusion in the second stage refined search, described below. In some cases $(\sim 10 \%)$, the best solutions formed isolated groups separated in density by more than $1000 \mathrm{~kg} \mathrm{~m}^{-3}$. We then worked with the best solution from each group. In all of these cases, only one of the groups survived this stage; the others were eliminated because of unphysical parameters.

\subsection{Refined search}

Before beginning a second, more focused search for the best model fits, we adjusted the best solution found during the broad search to better fit the photometry and trajectory data. We forward modelled the grain distribution and slightly changed the other free parameters until the best possible agreement was obtained. This is similar to the procedure usually followed in meteor modelling: the advantage of our methodology is that we had starting values for each parameter and we were able to rule out other local minima as not producing good solutions based on $\chi_{\mathrm{c}}^{2}$ values. 


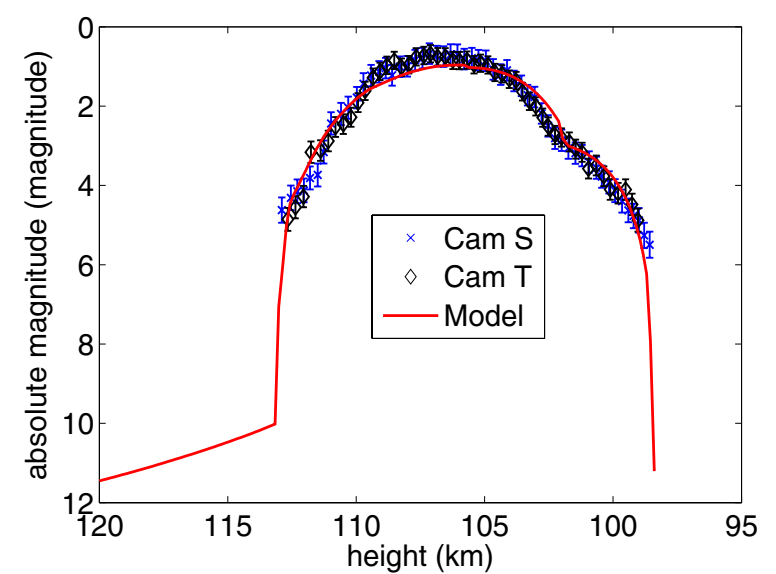

Fig. 6. The best lightcurve fit for the meteor 20060503-091349. The corresponding density is $970 \mathrm{~kg} \mathrm{~m}^{-3}$.

Table 4. Grain distribution found from modelling for meteor 20060503 091349 for the broad search and as determined through forward modelling for the refined search.

\begin{tabular}{cccc}
\hline \hline \multicolumn{2}{c}{ Broad search } & \multicolumn{2}{c}{ Refined search } \\
\# grains & mass of each grain & \# grains & mass of each grain \\
\hline 39278 & $6.60 \times 10^{-11}$ & 75767 & $0.93 \times 10^{-11}$ \\
15358 & $1.11 \times 10^{-10}$ & 55130 & $1.11 \times 10^{-10}$ \\
10146 & $1.40 \times 10^{-10}$ & 1198 & $1.15 \times 10^{-09}$ \\
\hline
\end{tabular}

Table 5. Best fit values of the free parameters from the broad search and for the final best solution for meteor 20060503-091349 from the refined search.

\begin{tabular}{lll}
\hline \hline Free parameter & Broad search & Refined search \\
\hline Heat of ablation $(\mathrm{J} / \mathrm{Kg})$ & $9.0 \times 10^{6}$ & $6.5 \times 10^{6}$ \\
Tlim $(\mathrm{K})$ & 1000 & 930 \\
Tboil $(\mathrm{K})$ & 1800 & 1900 \\
Specific heat $(\mathrm{J} / \mathrm{Kg} \mathrm{K})$ & 1000 & 1400 \\
Molar mass $($ atomic unit) & 36 & 36 \\
Thermal Conduct $(\mathrm{J} / \mathrm{m} \mathrm{s}$ K or W/m K) & 0.5 & 0.5 \\
\hline
\end{tabular}

For meteor 20060503-091349, the best model solution is shown in Fig. 6 for the lightcurve and for the deceleration in Fig. 7. The best fit grain distribution for this event is given in Table 4. The corresponding density is $970 \mathrm{~kg} \mathrm{~m}^{-3}$ and the values of other free parameters are given in Table 5.

Using this approach we obtained the best fit density for each meteor, but we proceed with another search of the parameter space to define the uncertainty range of this best fit density value. This time, we use a narrower range of densities, clustered around the best fit, and a higher search resolution (steps of $100 \mathrm{~kg} \mathrm{~m}^{-3}$ ). All solutions from this final search with $\chi_{\mathrm{c}}^{2} \leq 1$ for both the lightcurve and trajectory were considered possible solutions for the meteoroid and define our uncertainty range in density.

The density distribution of meteor 20060503-091349 (Fig. 8) suggests that the lowest possible density is $570 \mathrm{~kg} \mathrm{~m}^{-3}$, and the highest is $1170 \mathrm{~kg}^{-3}$. Using our methodology we find that meteor 20060503-091349 had a density of $970_{-400}^{+200} \mathrm{~kg} \mathrm{~m}^{-3}$. This meteor was photometrically saturated, and when we ran the model on the uncorrected lightcurve, the density was $480_{-130}^{+170} \mathrm{~kg} \mathrm{~m}^{-3}$, demonstrating the importance of including saturation corrections fully in meteor photometry (Kikwaya et al. 2010).

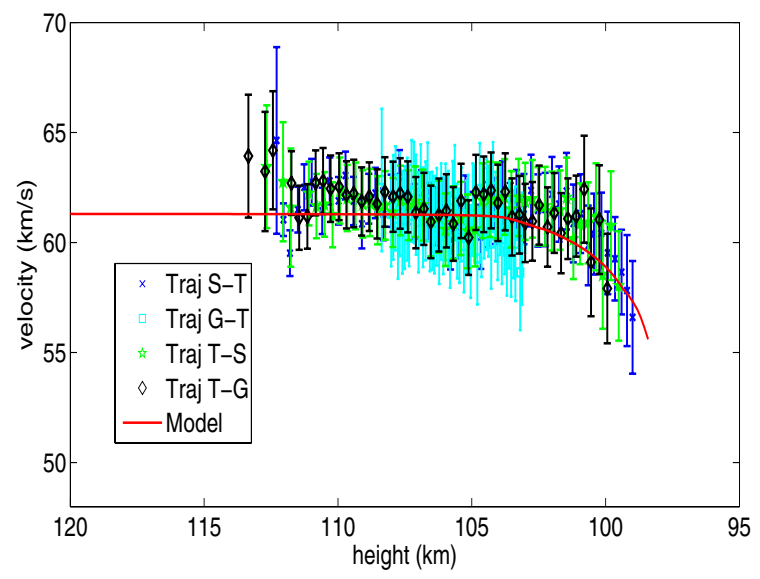

Fig. 7. The best trajectory fit for the meteor 20060503-091349 (see Fig. 2 for legend).

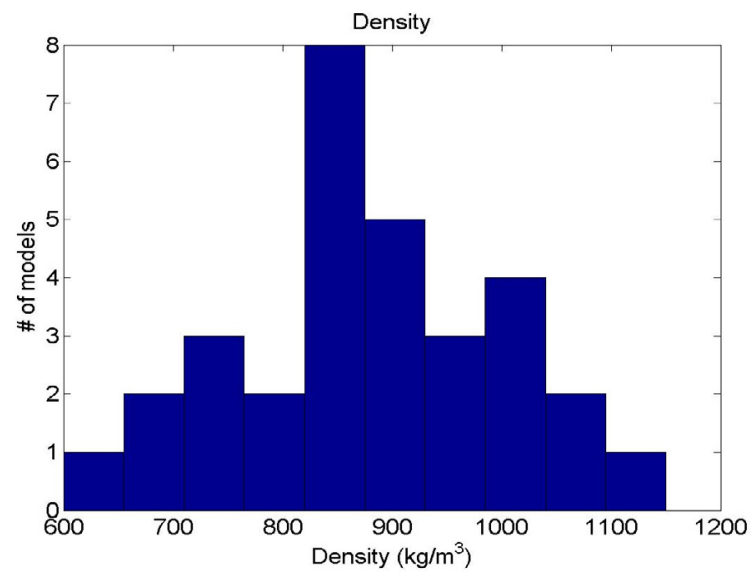

Fig. 8. The distribution of density solutions with $\chi_{\mathrm{c}}^{2} \leq 1$ for meteor 20060503-091349. The best solution is $970_{-400}^{+200} \mathrm{~kg} \mathrm{~m}^{-3}$.

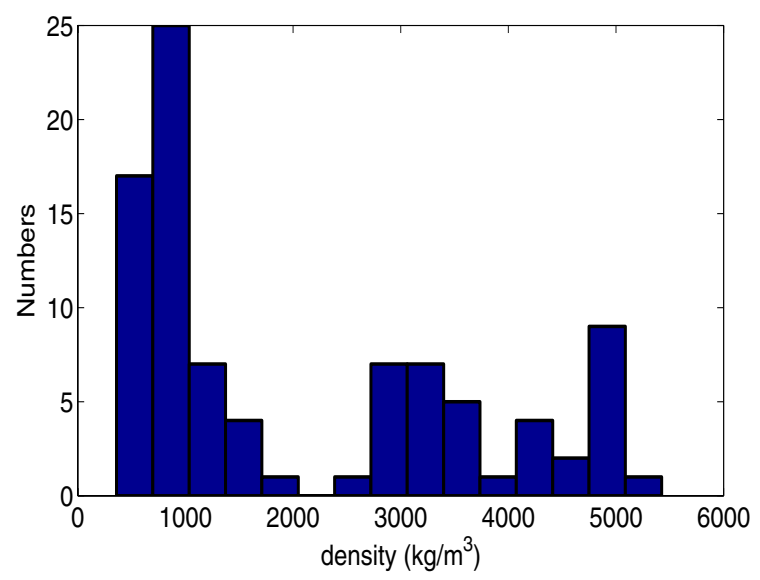

Fig. 9. Histogram showing the distribution of the best fit value for our meteoroid densities from our model fit to data (92 meteoroids total).

\section{Results and discussion}

\subsection{Origin of our meteoroids}

Densities were computed for all 92 meteors. The densities ranged from $360_{-100}^{+400} \mathrm{~kg} \mathrm{~m}^{-3}$ to $5425_{-800}^{+500} \mathrm{~kg} \mathrm{~m}^{-3}$. The distribution of densities (Fig. 9) shows three peaks (though the number statistics are low): one around $1000 \mathrm{~kg} \mathrm{~m}^{-3}$, one around $3000 \mathrm{~kg} \mathrm{~m}^{-3}$ and one around $5000 \mathrm{~kg} \mathrm{~m}^{-3}$. 
Figure 9 suggests a major population we interpret to be meteoroids rich in some form of Iron with densities ranging from $4150 \mathrm{~kg} \mathrm{~m}^{-3}$ to $5425 \mathrm{~kg} \mathrm{~m}^{-3}$. We make this interpretation following the study of Borovicka et al. (2005), who found a high percentage of meteoroids on Apollo-type orbits to have spectra indicating high Fe abundance; our bulk density measurements support this interpretation. A second population with average bulk density around $3000 \mathrm{~kg} \mathrm{~m}^{-3}$ we interpret to be similar in structure to chondrites based on similarity of these density values to the measured bulk density of recovered chondritic meteorites (cf. Consolmagno \& Britt 1998). This population has orbits which are like Jupiter-family comets (JFC) and also span Apollo-type orbits, suggestive of a main asteroid belt origin. By far our best represented population are meteoroids with relatively low density and Halley-type (HTC) or Nearly isotropic comets (NIC) - these represent $55 \%$ of our entire sample and range in density from $360 \mathrm{~kg} \mathrm{~m}^{-3}$ to $1510 \mathrm{~kg} \mathrm{~m}^{-3}$. One of the most interesting results of our study is the apparent gap around $2000 \mathrm{~kg} \mathrm{~m}^{-3}$ - this suggests that our sample does not contain many meteoroids with bulk proprieties similar to carbonaceous chondrites. We caution that these relative population sizes should not be treated as representative of the true mass-limited population weightings as the HTC/NICs have higher impact velocities and hence we see "deeper" into the mass distribution for HTC/NIC meteoroid population relative to the JFC/asteroidal population.

To quantify the likely origin for our mm-sized particles, as a first step we can use the Tisserand parameter relative to Jupiter to discriminate between meteoroids of Jupiter-family comet/Halley-type/nearly isotropic comet origin or asteroidal origin (Levison 1996; Weissman et al. 2002; Borovicka et al. 2005; Christou 2009).

The Tisserand parameter is given by:

$T_{\mathrm{j}}=\left(\frac{a_{\mathrm{j}}}{a}\right)+2 \sqrt{\left(\frac{a}{a_{\mathrm{j}}}\right)\left(1-e^{2}\right)} \cos (i)$

where $a_{\mathrm{j}}$ is the semimajor axis of Jupiter, $a$ is the semimajor axis of the meteoroid, and $i$ and $e$ are respectively the inclination and the eccentricity of the meteoroid's orbit. In broad terms, a Tisserand parameter less than 2 is characteristic of Halley type comets (originating in the Oort cloud), $2 \leq T_{\mathrm{j}}<3$ is characteristic of Jupiter family comets (originating in the Kuiper belt) and Tisserand parameters greater than 3 are typical of asteroidal orbits. This classification works for bodies which are subject only to gravitational perturbations and whose orbits are dominated by Jupiter. Both are good approximations at our large particle sizes as the Poynting-Robertson lifetime for mm-sized particles is two orders of magnitude larger than expected collisional lifetime (Grun et al. 1985). Further evidence that our meteoroid sample is largely unaffected by Poynting-Robertson drag is the lack of low eccentricity orbits, despite the fact that such orbits have much higher collisional probabilities with the Earth.

Of our sample, 51 meteoroids $(55 \%)$ had $T_{\mathrm{j}}<2,17$ meteoroids (19\%) had $T_{\mathrm{J}}$ between 2 and 3, and 24 meteoroids (26\%) had a $T_{\mathrm{J}}>3$ (Fig. 11). Two of our meteoroids (20070420082356 and 20080911-075207)had nominally hyperbolic velocities based on our procedure for computing initial velocity, but we observe that the hyperbolic excess was less than the spread in initial velocity computed between all three cameras in both cases. As both had low $\left(<1000 \mathrm{~kg} \mathrm{~m}^{-3}\right)$ bulk density and were likely cometary we omit them from further analysis noting that their inclusion to the large HTC/NIC meteoroid sample would change none of the conclusions which follow.
It must be noted that the Tisserand parameter cannot unambiguously discriminate these categories. There is some overlap close to the boundaries; also, a few asteroids (sometimes called Damocloids) (see Borovicka et al. 2005) are on Halley-type orbits, and some comets (notably 2P/Encke) have asteroidallike orbits.

In our data comparing density with orbit-type, the clearest division is between the HTC/NIC meteoroids and the objects on JFC or Asteroid-type orbits; meteoroids with $T_{\mathrm{j}}<2$ consistently have the lowest densities (smaller than $2000 \mathrm{~kg} \mathrm{~m}^{-3}$ in all cases), while those with $T_{\mathrm{j}}>2$ had densities greater than $2500 \mathrm{~kg} \mathrm{~m}^{-3}$. The division between Jupiter family comets and asteroids is less clear; while all high-density objects (densities greater than $4500 \mathrm{~kg} \mathrm{~m}^{-3}$ ) had $T_{\mathrm{j}}>3$, objects of intermediate density were found in both categories. This is similar to the distinct albedo distribution versus $T_{\mathrm{j}}$ found by Fernandez et al. (2001). They suggest that an old comet that loses all of its available volatiles or is covered by a mantle that prevents sublimation of subsurface ice will appear observationally as a near-Earth asteroid (NEA) or unusual asteroid (UA) (see Fig. 10). The dynamical lifetime of short-period comets being about 10-100 times longer than the devolatilization timescale (Levison \& Duncan 1994) allows one to observe extinct comets (as near-Earth asteroids or unusual asteroids) before they can disintegrate or collide with a planet. However, while Fernandez et al. (2006) finds that the albedo distribution of JFCs match HTCs and NICs well, but are distinct from asteroidal objects, we find the opposite trend with density, i.e. JFC related meteoroids appear to have nearly chondritic bulk density and are not low density cometary objects which we find exclusively with HTC and NIC-type meteoroids.

The most complete study of compositional variations among meteoroids at our sizes was reported by Borovicka et al. (2005), who in studying the orbital distribution of meteors with different spectral characterizations, used three more parameters to classify meteoroid orbits: perihelion distance, $q$, aphelion distance, $Q$, and inclination, $i$. Asteroids have aphelia inside Jupiter's orbit, while most comets have aphelia outside (see, for example, Fig. 12 in Borovicka et al. 2005), although there are exceptions. High inclination objects tend to be cometary, while low inclination objects may be asteroids or prograde comets.

Borovicka et al. (2005) divided meteoroid orbits into 5 classes based on a priori expected physical differences:

1. (SA) Sun-approaching orbits: $q<0.2$ AU.

2. (ES) Ecliptic shower orbits: meteor showers having orbits on the boundary of the Jupiter family and asteroidal objects; some of these would be classified as asteroidal though there are some clearly cometary parent bodies.

3. (HT) Halley-type orbits: $T_{\mathrm{j}}<2$ or $2<T_{\mathrm{j}}<3$ and $i>45^{\circ}$.

4. (JF) Jupiter family orbits: $2<T_{\mathrm{j}}<3$ and $i<45^{\circ}$ and $Q>$ 4.5 AU.

5. (A-C) Asteroidal-chondritic orbits: $T_{\mathrm{j}}>3$ or $Q<4.5 \mathrm{AU}$.

We use Borovicka et al.'s (2005) populations to examine our data and relate his compositional findings with our bulk density measurements. We found 9 meteoroids ( $\sim 10 \%$ of the total sample) that belong to the class of Sun-approaching orbits (SA) (Fig. 12). Borovicka et al. (2005) separated these meteoroids into a distinct class because they were, as a group, highly depleted in sodium compared to normal meteor spectra. The authors attribute this to thermal processing of the meteoroids when they are close to the sun. Although we cannot judge the sodium content of our meteors, it would be plausible to expect trends in density with perihelion distance, since thermal processing may include 
Table 6. All nine meteoroids of the Sun-approaching orbit class and values of their different parameters: photometric mass, perihelion, semi-major axis, eccentricity, inclination, pre-atmopheric velocity, Tisserand parameter, and bulk density.

\begin{tabular}{ccccccccc}
\hline \hline Code & $\begin{array}{c}\text { Phot mass } \\
(\mathrm{kg})\end{array}$ & $\begin{array}{c}q \\
(\mathrm{AU})\end{array}$ & $\begin{array}{c}a \\
(\mathrm{AU})\end{array}$ & $e$ & $\begin{array}{c}i \\
(\mathrm{o})\end{array}$ & $\begin{array}{c}v_{\infty} \\
\left(\mathrm{km} \mathrm{s}^{-1}\right)\end{array}$ & $\begin{array}{c}T_{\mathrm{j}} \\
\text { Density } \\
\mathrm{kg} \mathrm{m}^{-3}\end{array}$ \\
\hline $20060504-093103$ & $(7.9 \pm 0.6) \times 10^{-6}$ & $0.051 \pm 0.002$ & $1.599 \pm 0.038$ & $0.968 \pm 0.001$ & $15.160 \pm 1.059$ & $41.2 \pm 0.6$ & $3.5 \pm 0.5$ & $3550_{-600}^{+400}$ \\
$20070813-065828$ & $(1.0 \pm 0.1) \times 10^{-5}$ & $0.137 \pm 0.003$ & $2.397 \pm 0.073$ & $0.943 \pm 0.001$ & $18.279 \pm 0.684$ & $38.8 \pm 0.6$ & $2.6 \pm 0.3$ & $3150_{-400}^{+800}$ \\
$20080910-052352$ & $(1.5 \pm 0.8) \times 10^{-5}$ & $0.112 \pm 0.015$ & $2.348 \pm 0.314$ & $0.952 \pm 0.004$ & $30.495 \pm 3.600$ & $40.7 \pm 0.7$ & $2.6 \pm 0.5$ & $3500_{-500}^{+400}$ \\
$20080910-053428$ & $(2.0 \pm 0.2) \times 10^{-6}$ & $0.132 \pm 0.007$ & $1.984 \pm 0.116$ & $0.933 \pm 0.002$ & $6.871 \pm 1.694$ & $37.6 \pm 0.3$ & $3.1 \pm 0.4$ & $4100_{-100}^{+900}$ \\
$20080911-094844$ & $(1.7 \pm 0.2) \times 10^{-6}$ & $0.104 \pm 0.011$ & $1.741 \pm 0.140$ & $0.940 \pm 0.002$ & $14.436 \pm 3.198$ & $38.0 \pm 0.7$ & $3.4 \pm 0.5$ & $3470_{-500}^{+600}$ \\
$20090825-053106$ & $(1.9 \pm 0.2) \times 10^{-6}$ & $0.098 \pm 0.007$ & $2.255 \pm 0.176$ & $0.957 \pm 0.003$ & $17.181 \pm 2.169$ & $40.2 \pm 0.9$ & $2.7 \pm 0.4$ & $3020_{-500}^{+600}$ \\
$20090825-065903$ & $(3.9 \pm 0.4) \times 10^{-6}$ & $0.162 \pm 0.004$ & $2.704 \pm 0.097$ & $0.940 \pm 0.002$ & $21.310 \pm 0.641$ & $38.6 \pm 0.5$ & $2.4 \pm 0.3$ & $2645_{-700}^{+300}$ \\
$20090825-061542$ & $(1.7 \pm 0.2) \times 10^{-6}$ & $0.160 \pm 0.006$ & $3.422 \pm 0.364$ & $0.953 \pm 0.005$ & $10.242 \pm 1.022$ & $38.9 \pm 0.4$ & $2.0 \pm 0.3$ & $925_{-400}^{+500}$ \\
$20090902-085534$ & $(1.6 \pm 0.2) \times 10^{-6}$ & $0.031 \pm 0.007$ & $1.734 \pm 0.161$ & $0.982 \pm 0.005$ & $152.186 \pm 8.482$ & $50.9 \pm 0.8$ & $2.8 \pm 0.5$ & $4495_{-600}^{+600}$ \\
\hline
\end{tabular}

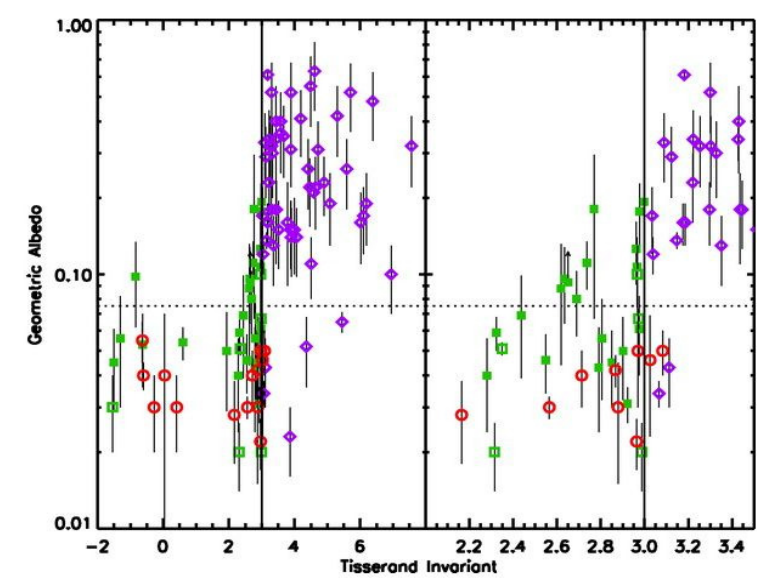

Fig. 10. Plot of known geometric albedos vs. Tisserand invariant for $T_{\mathrm{j}}<3$ NEAs and UAs (green squares) and $T_{\mathrm{j}}>3$ NEAs and UAs (purple diamonds). Active cometary nuclei are represented by red circles. A vertical line marks the dynamical boundary $T_{\mathrm{j}}=3$. There are 57 high-Tisserand albedos plotted, 56 of which are NEAs. The left panel shows the entire range of Tisserand values, and the right panel shows just the region near $T_{\mathrm{j}}=3$. The fraction of objects with comet-like albedos differs greatly from one side of the dynamical boundary to the other. A large fraction of the objects with $T_{\mathrm{j}}<3$ or precisely with $T_{\mathrm{j}}$ between 2 and 3 have comet-like albedos (from Fernandez et al. 2001).

sintering of the meteoroid material, with meteoroids becoming more compact as volatiles are lost or altered. Of the 9 meteors in the SA group, 6 have Tisserand parameters between 2 and 3, and 3 have Tisserand parameters above 3 ; none of the meteoroids with Tisserand parameter less than 2 are found in the SA group. The density of meteoroids belonging to this group in our sample ranges from $\sim 1000 \mathrm{~kg} \mathrm{~m}^{-3}$ to $\sim 4000 \mathrm{~kg} \mathrm{~m}^{-3}$ (Table 6), but most of the low- $q$ meteoroids have higher $\left(>2500 \mathrm{~kg} \mathrm{~m}^{-3}\right)$ densities. Whether this is due to their parent bodies or to thermal effects is not clear. Our results are consistent with the notion that low $q$ meteoroids are sintered, but small number statistics prevents us from stating this as a strong conclusion.

The inclinations of meteoroid orbits in the SA group are all close to the ecliptic, except for meteoroid 20090902-085534, with an inclination approaching $150^{\circ}$. From its aphelion $Q=$ 3.4 , the orbit of the meteoroid 20090902-085534 is inside Jupiter. It looks very much like a Halley type orbit (high inclination), possibly with a reduced $a$ due to a high ejection velocity from the sun-approaching parent comet when near perihelion. Surprisingly, this meteoroid has one of the highest densities in

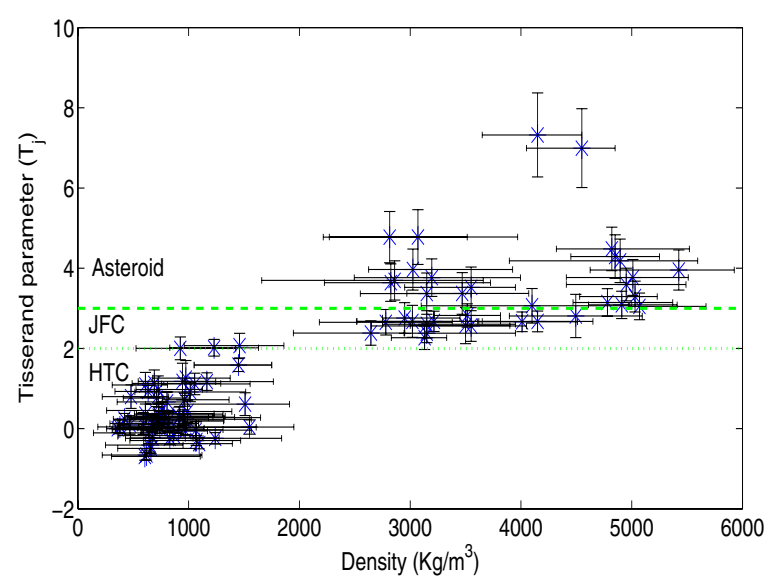

Fig. 11. Tisserand parameter versus density for our sample of meteoroids. Two lines are drawn at $T_{\mathrm{j}}=2$ and $T_{\mathrm{j}}=3$ to mark clearly the boundaries of different dynamical classes following Levison (1996).

our sample $\left(4495 \pm 600 \mathrm{~kg} \mathrm{~m}^{-3}\right)$, but is highly unlikely to be of asteroidal origin, since it is dynamically nearly impossible for asteroidal orbits to reach such high inclination. It may be an iron-rich inclusion from a Halley type comet, or a thermally processed meteoroid which has lost its volatiles and been sintered.

Our sample contained 24 meteoroids falling into Borovicka's Asteroid-chondritic class (Table 7), five of which overlap with the SA group. The average density of meteoroids in this class is $4100 \mathrm{~kg} \mathrm{~m}^{-3}$ (see Fig. 13) with a wide spread from 3000-5000. Of the 24 meteors, 22 satisfy the criteria because of their Tisserand parameter $\left(T_{\mathrm{j}}>3\right)$, and the other two because of their small $(Q<4.5 \mathrm{AU})$ aphelia.

In our observations, $55 \%$ of meteoroids fall into the HT class of high-inclination, cometary orbits (Fig. 14). Most of these meteoroids have low densities, with a minimum density of $360_{-100}^{+400} \mathrm{~kg} \mathrm{~m}^{-3}$ and maximum density of $1510_{-900}^{+400} \mathrm{~kg} \mathrm{~m}^{-3}$. There is one exception: the previously mentioned meteoroid 20090902-085534, with an unambiguously cometary orbit but a very high density. Its perihelion is much smaller than the other HT meteoroids, which may explain its very different physical structure.

The fourth Borovicka class (Jupiter family orbits), are lowinclination cometary orbits. We have 13 of these in our sample, including a 4th which overlaps with the SA class. The average density of JF meteoroids in our sample is $3190_{-480}^{+490} \mathrm{~kg} \mathrm{~m}^{-3}$, suggesting bulk properties akin to ordinary chondrites with low 
Table 7. All 24 meteoroids with Asteroidal-chondritic orbits (AC) (including 5 which overlap with the SA class and 4 with the ES class) and values of their different parameters: photometric mass, aphelion,semi-major axis, eccentricity, inclination, pre-atmopheric velocity, Tisserand parameter, density, and class.

\begin{tabular}{|c|c|c|c|c|c|c|c|c|c|}
\hline Code & $\begin{array}{l}\text { Photo mass } \\
(\mathrm{kg})\end{array}$ & $\begin{array}{c}Q \\
(\mathrm{AU})\end{array}$ & $\begin{array}{c}a \\
(\mathrm{AU})\end{array}$ & $e$ & $\begin{array}{l}i \\
\text { (०) }\end{array}$ & $\begin{array}{c}v_{\infty} \\
\left(\mathrm{km} \mathrm{s}^{-1}\right)\end{array}$ & $T_{\mathrm{j}}$ & $\begin{array}{l}\text { Density } \\
\mathrm{kg} \mathrm{m}^{-3}\end{array}$ & Class \\
\hline $20060504-093103$ & $(7.9 \pm 0.6) \times 10^{-6}$ & $147 \pm 0.076$ & $599 \pm 0.038$ & $68 \pm 0.001$ & $5.160 \pm 1.059$ & $41.20 \pm 0.61$ & $3.522 \pm 0.507$ & $3550_{-600}^{+400}$ & $\mathrm{AC} / \mathrm{SA}$ \\
\hline $20060505-102944$ & $(2.0 \pm 0.2) \times 10^{-5}$ & $1.187 \pm 0.013$ & $0.818 \pm 0.013$ & $0.452 \pm 0.012$ & $27.281 \pm 0.812$ & $20.60 \pm 0.25$ & $6.994 \pm 0.984$ & $4550_{-500}^{+300}$ & $\mathrm{AC}$ \\
\hline $20080910-053428$ & $(2.0 \pm 0.1) \times 10^{-6}$ & $3.837 \pm 0.226$ & $1.984 \pm 0.116$ & $0.933 \pm 0.002$ & $6.871 \pm 1.694$ & $37.60 \pm 0.33$ & $3.063 \pm 0.432$ & $4100_{-1000}^{+900}$ & $\mathrm{AC} / \mathrm{SA}$ \\
\hline $20080911-071428$ & $(1.1 \pm 0.1) \times 10^{-5}$ & $1.312 \pm 0.017$ & $0.767 \pm 0.011$ & $0.711 \pm 0.010$ & $5.768 \pm 1.091$ & $22.80 \pm 0.43$ & $7.323 \pm 1.048$ & $4150_{-500}^{+400}$ & $\mathrm{AC}$ \\
\hline 20080911-084529 & $(2.2 \pm 0.2) \times 10^{-6}$ & $3.487 \pm 0.355$ & $1.856 \pm 0.187$ & $0.878 \pm 0.004$ & $10.217 \pm 2.478$ & $33.50 \pm 0.43$ & $3.366 \pm 0.515$ & $3150_{-600}^{+400}$ & $\mathrm{AC}$ \\
\hline 20080911-094844 & $(1.7 \pm 0.1) \times 10^{-6}$ & $3.378 \pm 0.269$ & $1.741 \pm 0.140$ & $0.940 \pm 0.002$ & $14.436 \pm 3.198$ & $38.00 \pm 0.67$ & $3.371 \pm 0.519$ & $3470_{-500}^{+600}$ & $\mathrm{AC} / \mathrm{SA}$ \\
\hline $20090825-033603$ & $(1.2 \pm 0.1) \times 10^{-6}$ & $3.178 \pm 0.080$ & $1.738 \pm 0.042$ & $0.829 \pm 0.002$ & $4.354 \pm 0.540$ & $30.90 \pm 0.26$ & $3.639 \pm 0.466$ & $2825_{-600}^{+900}$ & $\mathrm{AC} / \mathrm{ES}$ \\
\hline $20090825-035228$ & $(2.9 \pm 0.2) \times 10^{-6}$ & $2.828 \pm 0.067$ & $1.578 \pm 0.034$ & $0.792 \pm 0.005$ & $5.756 \pm 0.398$ & $29.20 \pm 0.40$ & $3.967 \pm 0.512$ & $3025_{-400}^{+900}$ & $\mathrm{AC} / \mathrm{ES}$ \\
\hline 20090825-053106 & $(2.0 \pm 0.1) \times 10^{-6}$ & $4.412 \pm 0.349$ & $2.255 \pm 0.176$ & $0.957 \pm 0.003$ & $17.181 \pm 2.169$ & $40.20 \pm 0.93$ & $2.674 \pm 0.398$ & $3020_{-500}^{+600}$ & $\mathrm{AC} / \mathrm{SA}$ \\
\hline 20090825-070044 & $(6.3 \pm 0.4) \times 10^{-7}$ & $2.630 \pm 0.061$ & $1.484 \pm 0.029$ & $0.772 \pm 0.007$ & $4.859 \pm 0.125$ & $28.10 \pm 0.96$ & $4.184 \pm 0.544$ & $4895_{-1000}^{+700}$ & $\mathrm{AC} / \mathrm{ES}$ \\
\hline $20090825-081927$ & $(8.0 \pm 0.4) \times 10^{-8}$ & $2.736 \pm 0.154$ & $1.679 \pm 0.084$ & $0.630 \pm 0.011$ & $14.171 \pm 0.787$ & $22.40 \pm 0.41$ & $3.955 \pm 0.501$ & $5425_{-800}^{+500}$ & $\mathrm{AC}$ \\
\hline $20090825-091030$ & $(8.7 \pm 0.6) \times 10^{-7}$ & $1.854 \pm 0.031$ & $1.179 \pm 0.017$ & $0.573 \pm 0.005$ & $62.338 \pm 0.429$ & $36.10 \pm 0.73$ & $4.777 \pm 0.682$ & $3070_{-800}^{+900}$ & $\mathrm{AC}$ \\
\hline $20090825-032616$ & $(4.3 \pm 0.2) \times 10^{-6}$ & $3.161 \pm 0.176$ & $2.050 \pm 0.088$ & $0.542 \pm 0.020$ & $0.901 \pm 0.344$ & $14.80 \pm 0.20$ & $3.593 \pm 0.405$ & $4950_{-540}^{+540}$ & $\mathrm{AC}$ \\
\hline $20090825-035145$ & $(1.4 \pm 0.1) \times 10^{-6}$ & $1.742 \pm 0.041$ & $1.261 \pm 0.023$ & $0.381 \pm 0.008$ & $44.584 \pm 0.399$ & $27.70 \pm 0.30$ & $4.775 \pm 0.639$ & $2815_{-600}^{+700}$ & $\mathrm{AC}$ \\
\hline $20090825-050631$ & $(1.3 \pm 0.1) \times 10^{-6}$ & $3.034 \pm 0.080$ & $1.726 \pm 0.040$ & $0.758 \pm 0.006$ & $3.881 \pm 0.338$ & $27.10 \pm 0.70$ & $3.765 \pm 0.469$ & $3195_{-700}^{+800}$ & $\mathrm{AC}$ \\
\hline 20090825-050904 & $(5.1 \pm 0.3) \times 10^{-6}$ & $1.969 \pm 0.067$ & $1.490 \pm 0.033$ & $0.322 \pm 0.015$ & $12.770 \pm 0.510$ & $13.80 \pm 0.20$ & $4.481 \pm 0.542$ & $4820_{-500}^{+700}$ & $\mathrm{AC}$ \\
\hline $20090825-060500$ & $(2.6 \pm 0.2) \times 10^{-6}$ & $3.113 \pm 0.149$ & $1.706 \pm 0.077$ & $0.824 \pm 0.007$ & $4.966 \pm 0.913$ & $30.50 \pm 0.30$ & $3.696 \pm 0.489$ & $2860_{-1200}^{+800}$ & $\mathrm{AC} / \mathrm{ES}$ \\
\hline 20090826-020835 & $(1.1 \pm 0.1) \times 10^{-6}$ & $3.887 \pm 0.248$ & $2.445 \pm 0.124$ & $0.590 \pm 0.021$ & $23.277 \pm 0.434$ & $19.20 \pm 0.30$ & $3.146 \pm 0.345$ & $4780_{-310}^{+590}$ & $\mathrm{AC}$ \\
\hline 20090902-085534 & $(1.6 \pm 0.2) \times 10^{-6}$ & $3.437 \pm 0.328$ & $1.734 \pm 0.161$ & $0.982 \pm 0.005$ & $152.186 \pm 8.482$ & $50.90 \pm 0.80$ & $2.808 \pm 0.539$ & $4910_{-300}^{+500}$ & $\mathrm{AC} / \mathrm{SA}$ \\
\hline 20090909-010643 & $(2.5 \pm 0.2) \times 10^{-6}$ & $4.212 \pm 0.264$ & $2.483 \pm 0.131$ & $0.696 \pm 0.017$ & $3.134 \pm 0.216$ & $20.60 \pm 0.30$ & $3.086 \pm 0.341$ & $4910_{-300}^{+500}$ & $\mathrm{AC}$ \\
\hline 20090909-012810 & $(2.2 \pm 0.2) \times 10^{-6}$ & $2.979 \pm 0.107$ & $1.819 \pm 0.054$ & $0.638 \pm 0.010$ & $4.336 \pm 0.362$ & $21.40 \pm 0.46$ & $3.769 \pm 0.448$ & $5010_{-600}^{+500}$ & $\mathrm{AC}$ \\
\hline 20090909-013647 & $(4.9 \pm 0.4) \times 10^{-6}$ & $3.674 \pm 0.366$ & $2.332 \pm 0.183$ & $0.575 \pm 0.034$ & $14.262 \pm 0.597$ & $16.10 \pm 0.40$ & $3.293 \pm 0.385$ & $5030_{-500}^{+200}$ & $\mathrm{AC}$ \\
\hline 20090911-021830 & $(1.5 \pm 0.1) \times 10^{-6}$ & $4.184 \pm 0.273$ & $2.544 \pm 0.136$ & $0.644 \pm 0.019$ & $20.443 \pm 0.392$ & $20.15 \pm 0.70$ & $3.048 \pm 0.333$ & $5070_{-100}^{+600}$ & $\mathrm{AC}$ \\
\hline 20090911-034442 & $(1.7 \pm 0.1) \times 10^{-6}$ & $2.472 \pm 0.072$ & $1.494 \pm 0.038$ & $0.655 \pm 0.007$ & $4.452 \pm 0.469$ & $23.40 \pm 0.63$ & $4.291 \pm 0.543$ & $4850_{-400}^{+400}$ & $\mathrm{AC}$ \\
\hline
\end{tabular}

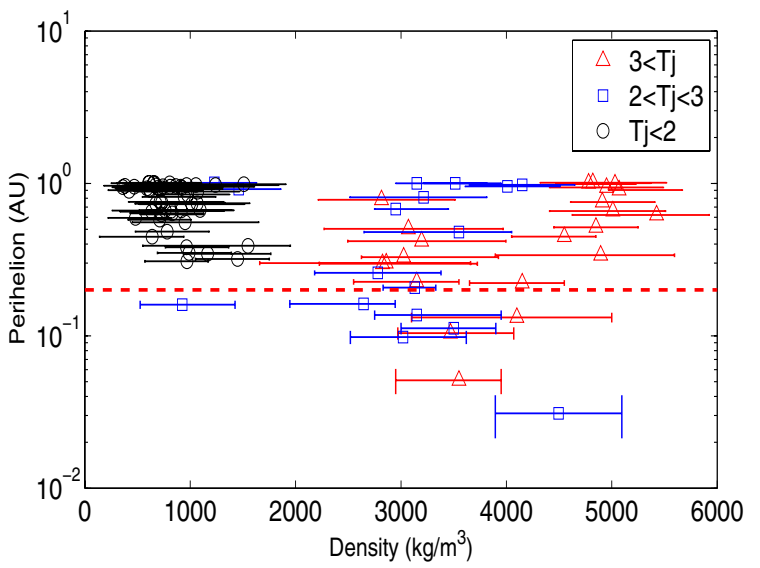

Fig. 12. The perihelion distance versus bulk density for our observed sample. The dashed line marks the boundary of meteoroid orbits with perihelion $(q)$ below 0.2 AU identified by Borovicka et al. (2005) as being in the class of Sun-approaching orbits.

porosity. One of these, meteor 20090825-061442, has a bulk density $(\rho)$ less than $1000 \mathrm{~kg} \mathrm{~m}^{-3}$, but also a low $T_{\mathrm{j}}$; within error it can be placed in the HTC (Halley type comets) class.

This is perhaps our most significant result. We are sampling from a range of original JF bodies (as the JFC meteoroids were observed from a wide range of radiants over the course of the year). JFCs are believed to be derived from the Kuiper-Belt

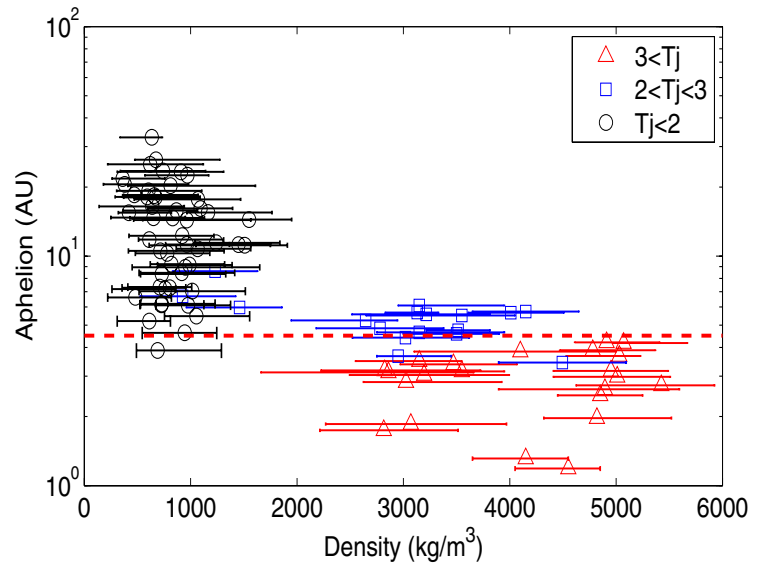

Fig. 13. Aphelion versus density for our meteoroid sample. The horizontal line indicates an aphelion $(Q)$ of $4.5 \mathrm{AU}$, below which meteoroids are expected to be of asteroidal origin following Borovicka et al. (2005).

and then been perturbed inward to become active comets, retaining their flat inclination distribution (cf. Fernandez 1980; and recent review by Morbidelli 2008). In this scenario JFCs form in the outer part of the solar system and are not likely collisional fragments of larger bodies - i.e. they are expected to be primitive. Prior to the sample return from $81 \mathrm{P} /$ Wild 2 , expectations were that most material in JFCs would resemble chondritic 


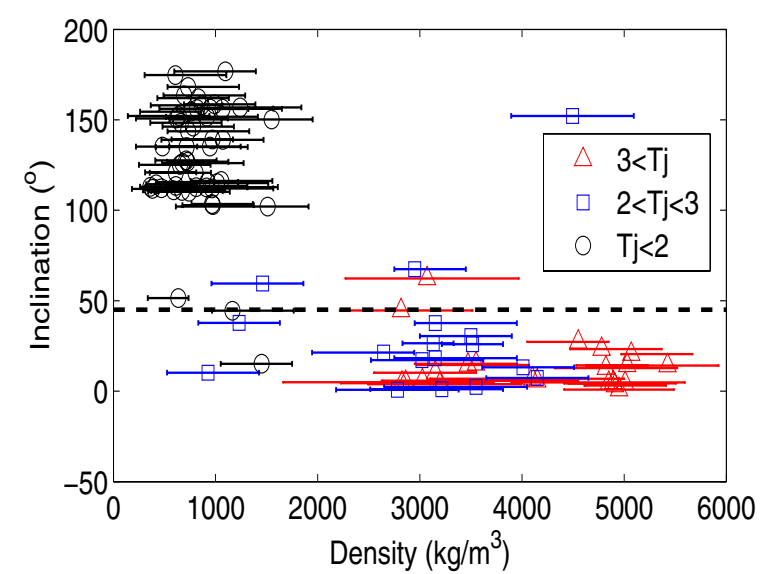

Fig. 14. The orbital inclination versus bulk density for our meteoroid sample. The horizontal dashed line marks the boundary of meteoroid orbits with inclination $(i)$ above $45^{\circ}$. Meteoroids with inclination $i>$ $45^{\circ}$ and $2<T_{\mathrm{j}}<3$ or $T_{\mathrm{j}}<2$ form the class of meteoroids with Halley type orbits (HT) according to the scheme of Borovicka et al. (2005).

porous (CP) IDPs in chemistry; instead analysis of the aerogelcaptured particles from Wild 2 has shown that a large fraction of this JFCs material is chondrite-like with abundant refractory material indicative of formation of some of Wild 2 in the inner solar system (Ishii et al. 2008; Brownlee et al. 2006). This refractory material was found from primary particles impacting aerogel which originally had sizes estimated to be in the tens of micron range in most cases (Horz et al. 2006).

Our findings suggest that the trend found for 81P/Wild 2 applies to other JFCs and extends the existence of refractory-rich particles in JFCs up to mm-sized particles which in our model are comprised of micron - tens of micron-sized grains. The implication is that the process which implanted refractory material in the JFC population was ubiquitous (and not confined to $81 \mathrm{P} /$ Wild 2). Furthermore, we find a clear distinction in the bulk density properties of cometary meteoroids originating from JFCs versus those from HTC/NICs suggesting different formation locations and/or evolutionary pathways.

In Borovicka's final class, the ecliptic showers, we found four meteoroids, all members of the Northern Iota Aquariids. If it were not for this class, all of these meteoroids would fall into the asteroidal class because of the large Tisserand parameter. The average density of these meteoroids is $3200 \mathrm{~kg} \mathrm{~m}^{-3}$, so these objects appear to be chondritic, similar to our findings for the Jupiter family material. The fact that, if not for their shower association, these meteoroids would be classified as asteroidal, suggests that many of the meteoroids in the asteroidal-chondritic class may actually be from Jupiter family comets. Indeed the NIA shower is thought to be part of the Taurid complex, linked to $2 \mathrm{P} /$ Encke (Brown et al. 2008).

\subsection{Meteoroid strength and density}

Meteor beginning height has been used in past work as a measure of the strength of meteoroids in the millimeter size range (see Ceplecha 1958, 1967, 1968, 1988; Borovicka et al. 2005). For two meteoroids having the same pre-atmospheric velocity, a stronger/denser meteoroid will start to ablate at a lower height than a weaker/low density meteroid. Figure 16 shows the beginning height versus density for the meteoroids in our sample, divided into five classes described in the previous section. The

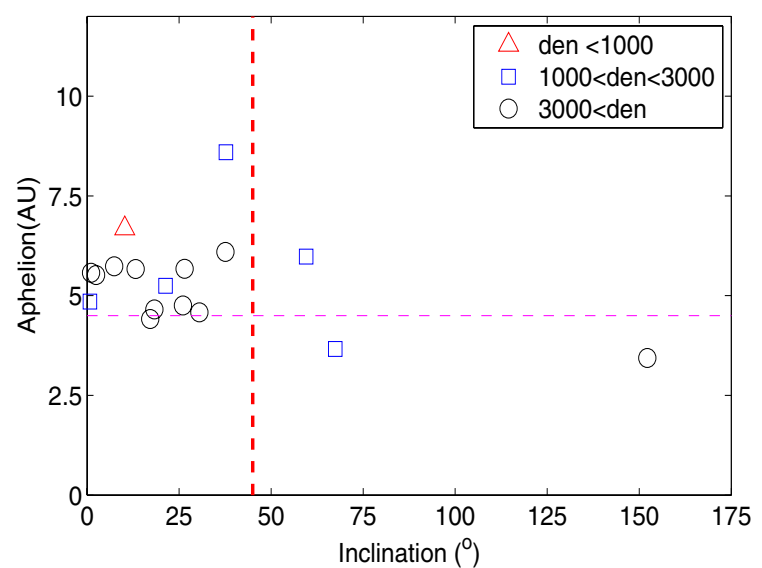

Fig. 15. Inclination versus aphelion for meteoroid orbits with Tisserand parameter $2<T_{\mathrm{j}}<3$ nominally in the JFC (Jupiter family comets) class. The vertical line denotes the boundary of meteoroid orbits with inclination $i<45$, and the horizontal line the boundary for meteoroid orbits with aphelion $Q>4.5 \mathrm{AU}$. These meteoroids constitute the class of Jupiter family orbits (see Borovicka et al. 2005).

figure clearly shows that meteoroids from the AC class, which have the highest density, start at lower heights, while the HT meteoroids, with low densities, have high beginning heights, trends entirely as expected.

We found that the beginning heights of meteoroids in our sample seem to be mass-independent. This is in agreement with the work done by Koten et al. (2004) on several meteor showers (see their Fig. 3 on beginning heights versus photometric mass of fainter Perseid meteors). For the size of meteoroids in our sample with an average mass around $\sim 10^{-6} \mathrm{~kg}$, as predicted by dust-ball model (Hawkes \& Jones 1975), there is no clear dependence between beginning heights and photometric mass. There is a clear relationship between beginning heights and velocity, as expected (see Fig. 19). HT meteoroids in our sample (low density material) have high velocity and start to ablate very high in the atmosphere, whereas AC meteoroids have lower beginning heights, but high densities, again as found in earlier works.

Figure 17 shows the plot of velocity versus density. The general trend of our plot is that high density meteoroids (AC) have small velocities, and low density meteoroids (HT) have high velocities as expected from their orbital properties and the conditions of encounter with the Earth. The only meteoroid with a large velocity and high density is meteor 20090902-085534, with a cometary, Sun-approaching orbit whose origin and probable history have already been discussed. We observe that the bulk density trends we see with velocity make clear that we are not biasing the measurements due to limitations of our measurements; in general one expects high velocity meteors to show the least deceleration and if we were not properly measuring deceleration (or it was below our measurement precision) our model would tend to show artificlally high bulk densities (all else being the same) which is the opposite of the trend we see.

\subsection{Comparison using the $K_{B}$ parameter}

Ceplecha introduced a parameter, $K_{\mathrm{B}}$, which classifies meteoroids according to their beginning height, while simultaneously taking into account their pre-atmospheric velocity and the trajectory slope. The parameter is based on the assumption that all meteoroids begin to be luminous at the same surface temperature and hence beginning height will be a strong function of 
Table 8. All thirteen meteoroids with Jupiter Family orbits (JF) (including 4 which overlap with the SA class) and values of their different parameters: photometric mass, aphelion,semi-major axis, eccentricity, inclination, pre-atmopheric velocity, Tisserand parameter, density, and class.

\begin{tabular}{|c|c|c|c|c|c|c|c|c|c|}
\hline Code & $\begin{array}{c}\text { Photo mass } \\
(\mathrm{kg})\end{array}$ & $\begin{array}{c}Q \\
(\mathrm{AU})\end{array}$ & $\begin{array}{c}a \\
(\mathrm{AU})\end{array}$ & $e$ & $\begin{array}{c}i \\
\text { (o) }\end{array}$ & $\begin{array}{c}v_{\infty} \\
\left(\mathrm{km} \mathrm{s}^{-1}\right)\end{array}$ & $T_{\mathrm{j}}$ & $\begin{array}{l}\text { Density } \\
\mathrm{kg} \mathrm{m}^{-3}\end{array}$ & Class \\
\hline $20070813-065828$ & $(1.0 \pm 0.1) \times 10^{-5}$ & $4.656 \pm 0.145$ & $97 \pm 0.073$ & $0.943 \pm 0.001$ & $.279 \pm 0.684$ & $38.8 \pm 0.6$ & $2.6 \pm 0.3$ & $3150_{-800}^{+400}$ & $\mathrm{JF} / \mathrm{SA}$ \\
\hline $20080910-052352$ & $(1.5 \pm 0.1) \times 10^{-5}$ & $4.583 \pm 0.616$ & $2.348 \pm 0.314$ & $0.952 \pm 0.004$ & $30.495 \pm 3.600$ & $40.7 \pm 0.7$ & $2.6 \pm 0.5$ & $3500_{-400}^{+500}$ & $\mathrm{JF} / \mathrm{SA}$ \\
\hline 20080910-064102 & $(5.2 \pm 0.4) \times 10^{-6}$ & $5.516 \pm 0.930$ & $2.997 \pm 0.474$ & $0.840 \pm 0.019$ & $2.392 \pm 1.539$ & $28.2 \pm 0.5$ & $2.6 \pm 0.4$ & $3550_{-900}^{+500}$ & $\mathrm{JF}$ \\
\hline $20090825-065903$ & $(3.9 \pm 0.4) \times 10^{-6}$ & $5.247 \pm 0.192$ & $2.704 \pm 0.097$ & $0.940 \pm 0.002$ & $21.310 \pm 0.641$ & $38.6 \pm 0.5$ & $2.4 \pm 0.3$ & $2645_{-300}^{+700}$ & $\mathrm{JF} / \mathrm{SA}$ \\
\hline $20090825-043435$ & $(4.8 \pm 0.5) \times 10^{-6}$ & $4.850 \pm 0.149$ & $2.555 \pm 0.076$ & $0.898 \pm 0.002$ & $0.767 \pm 0.437$ & $34.2 \pm 0.3$ & $2.7 \pm 0.3$ & $2780_{-600}^{+600}$ & $\mathrm{JF}$ \\
\hline $20090820-014058$ & $(5.0 \pm 0.4) \times 10^{-6}$ & $6.097 \pm 0.339$ & $3.554 \pm 0.169$ & $0.716 \pm 0.014$ & $37.598 \pm 0.248$ & $26.2 \pm 0.5$ & $2.4 \pm 0.2$ & $3150_{-200}^{+800}$ & $\mathrm{JF}$ \\
\hline $20090825-034528$ & $(1.1 \pm 0.7) \times 10^{-6}$ & $5.737 \pm 0.439$ & $3.357 \pm 0.219$ & $0.709 \pm 0.019$ & $7.325 \pm 0.245$ & $15.7 \pm 0.3$ & $2.7 \pm 0.3$ & $4150_{-500}^{+500}$ & $\mathrm{JF}$ \\
\hline $20090825-061542$ & $(1.7 \pm 0.2) \times 10^{-6}$ & $6.684 \pm 0.728$ & $3.422 \pm 0.364$ & $0.953 \pm 0.005$ & $10.242 \pm 1.022$ & $38.9 \pm 0.4$ & $2.1 \pm 0.3$ & $925_{-500}^{+400}$ & $\mathrm{JF} / \mathrm{SA}$ \\
\hline $20090825-070933$ & $(3.1 \pm 0.2) \times 10^{-6}$ & $5.572 \pm 0.157$ & $3.191 \pm 0.079$ & $0.746 \pm 0.006$ & $1.064 \pm 0.122$ & $19.6 \pm 0.4$ & $2.7 \pm 0.3$ & $3215_{-700}^{+600}$ & $\mathrm{JF}$ \\
\hline $20090902-084143$ & $(6.3 \pm 0.5) \times 10^{-6}$ & $8.595 \pm 1.091$ & $4.801 \pm 0.545$ & $0.790 \pm 0.024$ & $37.713 \pm 0.513$ & $26.7 \pm 0.7$ & $2.0 \pm 0.2$ & $1230_{-400}^{+400}$ & $\mathrm{JF}$ \\
\hline $20090911-030523$ & $(4.8 \pm 0.6) \times 10^{-6}$ & $5.675 \pm 0.357$ & $2.941 \pm 0.178$ & $0.929 \pm 0.005$ & $26.498 \pm 0.526$ & $38.2 \pm 0.3$ & $2.3 \pm 0.3$ & $3130_{-300}^{+200}$ & $\mathrm{JF}$ \\
\hline 20090911-035942 & $(1.4 \pm 0.2) \times 10^{-6}$ & $4.756 \pm 0.273$ & $2.879 \pm 0.137$ & $0.652 \pm 0.017$ & $26.004 \pm 0.319$ & $20.6 \pm 0.2$ & $2.8 \pm 0.3$ & $3515_{-300}^{+300}$ & $\mathrm{JF}$ \\
\hline 20090911-040433 & $(2.3 \pm 0.2) \times 10^{-5}$ & $5.670 \pm 0.203$ & $3.313 \pm 0.101$ & $0.711 \pm 0.009$ & $13.201 \pm 0.157$ & $17.4 \pm 0.3$ & $2.7 \pm 0.2$ & $4010_{-400}^{+500}$ & JF \\
\hline
\end{tabular}

Table 9. All 4 meteoroids of the Ecliptic shower orbits class (ES) and values of their different parameters: photometric mass, perihelion, semi-major axis, eccentricity, inclination, pre-atmopheric velocity, Tisserand parameter, and density.

\begin{tabular}{ccccccccc}
\hline \hline Code & $\begin{array}{c}\text { Photom. mass } \\
(\mathrm{kg})\end{array}$ & $\begin{array}{c}q \\
(\mathrm{AU})\end{array}$ & $\begin{array}{c}a \\
(\mathrm{AU})\end{array}$ & $e$ & $\begin{array}{c}i \\
(\circ)\end{array}$ & $\begin{array}{c}v_{\infty} \\
\left(\mathrm{km} \mathrm{s}^{-1}\right)\end{array}$ & $\begin{array}{c}T_{\mathrm{j}} \\
\text { Density } \\
\mathrm{kg} \mathrm{m}^{-3}\end{array}$ \\
\hline 20090825033603 & $(1.2 \pm 0.1) \times 10^{-6}$ & $0.297 \pm 0.005$ & $1.738 \pm 0.042$ & $0.829 \pm 0.002$ & $4.354 \pm 0.540$ & $30.90 \pm 0.26$ & $3.639 \pm 0.5$ & $2825_{-900}^{+600}$ \\
20090825035228 & $(2.9 \pm 0.2) \times 10^{-6}$ & $0.328 \pm 0.005$ & $1.578 \pm 0.034$ & $0.792 \pm 0.005$ & $5.756 \pm 0.398$ & $29.20 \pm 0.40$ & $3.967 \pm 0.5$ & $3025_{-900}^{+400}$ \\
20090825070044 & $(6.3 \pm 0.4) \times 10^{-7}$ & $0.338 \pm 0.004$ & $1.484 \pm 0.029$ & $0.772 \pm 0.007$ & $4.859 \pm 0.125$ & $28.10 \pm 0.96$ & $4.184 \pm 0.5$ & $4895_{-700}^{+1000}$ \\
20090825060500 & $(2.6 \pm 0.2) \times 10^{-6}$ & $0.300 \pm 0.009$ & $1.706 \pm 0.077$ & $0.824 \pm 0.007$ & $4.966 \pm 0.913$ & $30.50 \pm 0.30$ & $3.696 \pm 0.5$ & $2860_{-800}^{+1200}$ \\
\hline
\end{tabular}

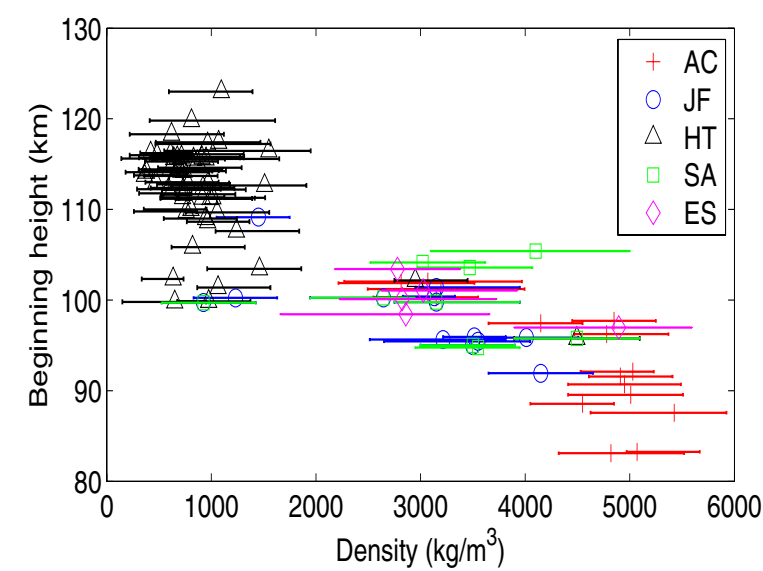

Fig. 16. Beginning height versus density of meteoroids of our sample. The meteoroids are divided according to the orbital classes defined above.

the meteoroid composition. Inclusion of velocity and entry angle helps to remove the dependence on geometry of the beginning height, and gives a parameter closely tied to the material strength of the meteoroid. $K_{\mathrm{B}}$ is defined as (Ceplecha 1967):

$K_{\mathrm{B}}=\log \rho_{\mathrm{B}}+2.5 \log v_{\infty}-0.5 \log \cos Z_{\mathrm{R}}$

where $\rho_{\mathrm{B}}$ is the air density at the beginning of the luminous trajectory, expressed in $\mathrm{g} \mathrm{cm}^{-3}, v_{\infty}$ is the initial velocity (pre- atmospheric velocity) expressed in $\mathrm{cm} \mathrm{s}^{-1}$, and $z_{\mathrm{R}}$ is the zenithal angle of the radiant. Ceplecha (1988) used the

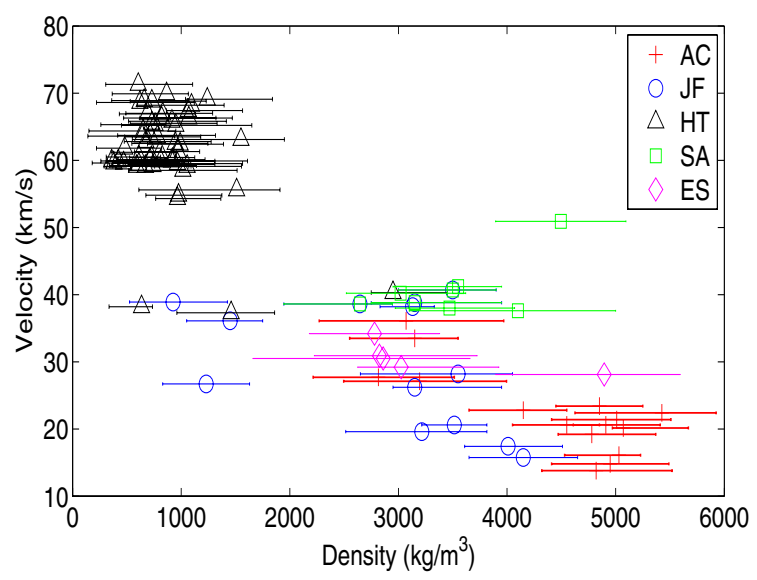

Fig. 17. Velocity versus density of meteoroids of our sample. The meteroids are divided according to the orbital classes defined above.

$K_{\mathrm{B}}$ parameter to separate meteoroids in the $\mathrm{mm}$ to $\mathrm{cm}$-size range into four populations:

1. group A: $7.30 \leq K_{\mathrm{B}}<8.00$;

2. group B: $7.10 \leq K_{\mathrm{B}}<7.30$

3. group C: $6.60 \leq K_{\mathrm{B}}<7.10$;

4. group D: $K_{\mathrm{B}}<6.60$.

He associated group A with ordinary chondrite material, group B with carbonaceous chondrites, group $\mathrm{C}$ with dense and regular cometary material, and D with weak cometary meteoroids (Ceplecha 1988). Later, Ceplecha (1988) also added a fifth 


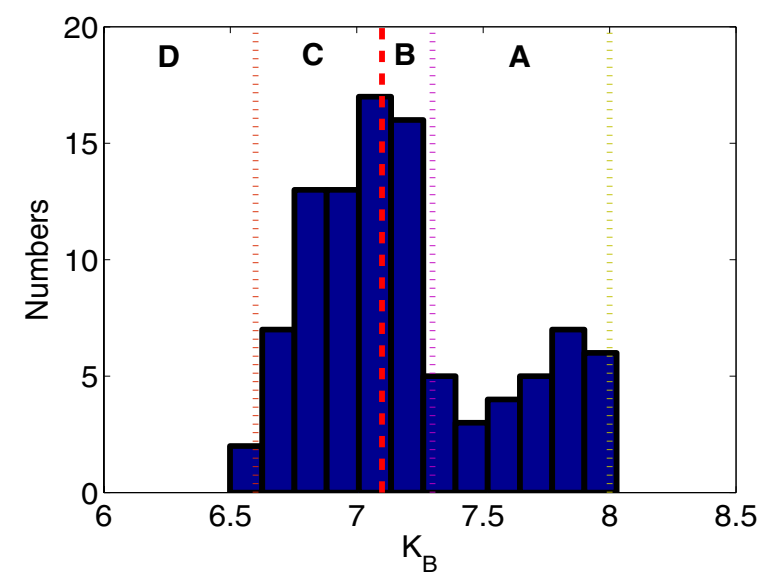

Fig. 18. Histogram of $K_{\mathrm{B}}$ values of our sample. Two groups are clearly distinguishable: group A around $K_{\mathrm{B}} \sim 7.8$ and group $\mathrm{C}$ around $K_{\mathrm{B}} \sim$ 7.0. The nominal population demarcations follow Ceplecha's (1967) bin sizes for each group but shifted by +0.18 .

group, Asteroidal, to the four mentioned here, for meteoroids with $K_{\mathrm{B}} \geq 8$. He also divided group $\mathrm{C}$ into three subgroups based on orbital elements, but due to our small number statistics, we treat these as one.

Equation (5) was developed and first applied to meteors photographed by Super-Schmidt cameras (see Ceplecha 1967, 1988; Bellot Rubio et al. 2002). For the fainter meteors observed by intensified video systems, the parameter $K_{\mathrm{B}}$ is calculated in the same way, but an offset of 0.15 is added (see Ceplecha 1988) because the $K_{\mathrm{B}}$ criterion is based on meteor beginning heights, which depend on the sensitivity of the camera. TV systems being more sensitive than Super-Schmidt cameras (see Sarma \& Jones 1985), the meteors will be observed higher up, and $K_{\mathrm{B}}$ will be systematically shifted to lower values.

Figure 18 shows a histogram of the $K_{\mathrm{B}}$ values for our sample using a nominal shift for our observations of +0.18 relative to that used by Ceplecha (1988). Two clear peaks are evident: one around $K_{\mathrm{B}} \sim 7.0$, likely corresponding to group $\mathrm{C}$; one around $K_{\mathrm{B}} \sim 7.8$, which we believe is actually the A group. We note that the general form of this distribution is similar to that found by Ceplecha (1967) for Super-Schmidt meteors, but our peaks are offset by 0.18 from the Ceplecha (1967) peaks. This is a larger shift than suggested for the TV data by Ceplecha (1988), but can be understood as the improvement in sensitivity of modern electro-optical system compared to the early ISIT systems used by Sarma \& Jones (1985). The peak of group D is not clearly visible, because only a few meteoroids in our sample belong to this group. There is no evidence for a distinct peak corresponding to group B in our data. Group C contains most of the meteoroids in our sample $(44 \%)$.

We can compare the average density of meteoroids in these groups found from our data to previous results. Table 10 summarizes the results from five studies, including ours. Three of the studies (Ceplecha 1966, 1967; Bellot Rubio et al. 2002) used Super-Schmidt data, for meteoroids slightly larger than those in our data set. Ceplecha finds densities for these meteoroids which are nearly twice those found by Bellot Rubio. The density calculation of Ceplecha (1967) is based on the Levin (1956) formula describing the meteoroid surface temperature $\tau(0, h)$ at height $h$. For the analysis by Ceplecha (1967), $\tau$ is calculated at the beginning height. Thus, Ceplecha's densities are averages, and not derived from individual fits to each meteor in his sample. Bellot Rubio et al. (2002) described the dynamical and photometric behavior of individual faint meteors observed with Super-Schmidt cameras by means of the single body theory to arrive at the densities values in Table 10. Our values are larger than these other studies for Groups A, and D which may reflect a physical change in meteoroid properties with mass or particular differences between our model approach and these works.

Although we have no meteoroids in Ceplecha's asteroidal class $\left(K_{\mathrm{B}} \geq 8\right)$, we may still have asteroidal material in group A, which overlaps significantly with Borovicka's A-C class. We do not find evidence for a distinct class $\mathrm{B}$, but rather there seems to be a continuum from class $\mathrm{A}$ to $\mathrm{C}$. We do find a very clear distinction between the various Ceplecha classes based on $K_{\mathrm{B}}$ and $T_{\mathrm{j}}$ and bulk density supporting the notion that physical differences are dominated by origin rather than evolution.

\subsection{Comparison of bulk density and meteoroid spectra}

Borovicka et al. (2005) has argued that the dependence of beginning height on speed is sufficient to recognize different material strength among different spectral classes of meteoroids. In their Fig. 15, they clearly show that the average beginning height of Na-free meteoroids and Iron meteoroids as being lower than other meteoroids. In contrast, the Fe-poor meteoroids tend to have higher beginning heights.

Figure 19 based on our data, shows the same relationship between beginning height and speed using the 4 classes identified by Borovicka et al. (2005). Figure 20 also shows the same relationship, but for the 4 groups according to Ceplecha's classification. Group A is clearly concentrated in the lower part of the thick line. There are 4 meteoroids of group A at high velocity. This might be smeared by the zenith angle of the radiant. Groups C and D are all concentrated in the upper part of the thick line, as expected. We found 5 meteoroids with beginning heights below $\sim 90 \mathrm{~km}$ (Fig. 19). They all belong to the AC (Asteroidchondritic) class and are likely iron-rich (following Borovicka et al. 2005), based on their density. The same meteoroids in Fig. 20 fall into the A-group, whose average density from our analysis is $3800 \pm 800 \mathrm{~kg} \mathrm{~m}^{-3}$ (see Table 10).

The sun-approaching meteoroids (SA class) with perihelion $q \leq 0.22$ AU have no sodium in their spectra according to Borovicka et al. (2005). In Fig. 20, these meteoroids appeared to be spread over three different groups (A-group, C-group and D-group). We cannot then attach to them a unique average density. This is a consequence of the fact that SA meteoroids evolve from different dynamical populations and hence likely have very different starting compositions, and structures. They all have thermal heating in common, but the final products are still quite different. Indeed, while most SA densities are high, supporting the notion that evolution dominates over origin for this population, at least one has a low density and probable HT origin suggesting this is not the case. Our Fe-poor meteoroids with higher beginning heights all can be easily assigned to the HT class (Halley type orbits) (Fig. 19). They also belong to the C-group which has an average bulk density of $1000 \mathrm{~kg} \mathrm{~m}^{-3}$. They are all likely cometary meteoroids.

\subsection{Interpretation of meteoroid physical properties}

In broad terms our meteoroid bulk density results can be broken down into a high density class (density $\geq 3000 \mathrm{~kg} \mathrm{~m}^{-3}$ ) and low density (density $\leq 2000 \mathrm{~kg} \mathrm{~m}^{-3}$ ) class. For the former particles, a high density is required for constiuent grains. Indeed, most of these particles have bulk densities reminiscent of chondritic 
Table 10. Average density in Ceplcha's four meteor classes from different studies.

\begin{tabular}{cccccc}
\hline \hline Work & Instruments & $\begin{array}{c}\text { Group A } \\
\text { density }\left(\mathrm{kg} \mathrm{m}^{-3}\right)\end{array}$ & $\begin{array}{c}\text { Group B } \\
\text { density }\left(\mathrm{kg} \mathrm{m}^{-3}\right)\end{array}$ & $\begin{array}{c}\text { Group C } \\
\text { density }\left(\mathrm{kg} \mathrm{m}^{-3}\right)\end{array}$ & $\begin{array}{c}\text { Group D } \\
\text { density }\left(\mathrm{kg} \mathrm{m}^{-3}\right)\end{array}$ \\
\hline Ceplecha (1966) & Photographic Super-Schmidt & 4000 & 2200 & 1400 & - \\
Ceplecha (1967) & Photographic Super-Schmidt & 4000 & 2200 & 1400 & - \\
Ceplecha (1988) (Table 6) & Photographic and TV & $1400-2700$ & $650-1700$ & $550-910$ & $180-380$ \\
Bellot Rubio et al. (2002) & Photographic Super-Schmidt & 2400 & 1400 & 400 & - \\
This work & TV systems & 3800 & - & 800 & - \\
\hline
\end{tabular}

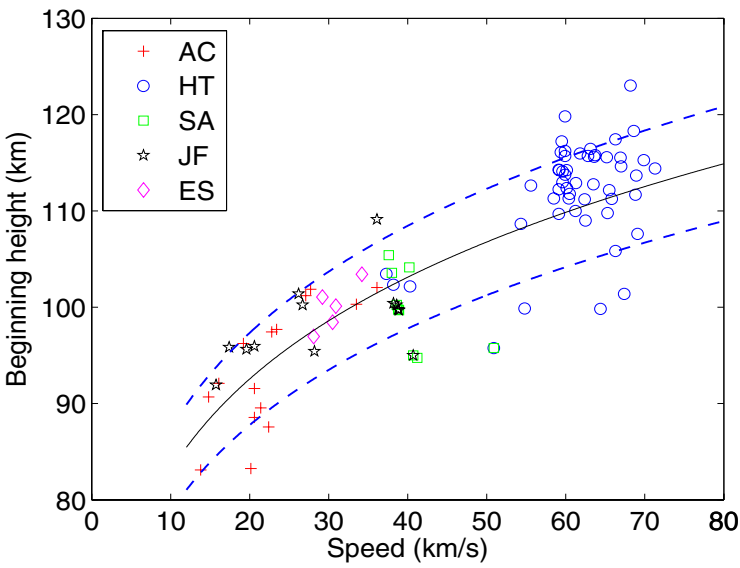

Fig. 19. Meteor beginning height as a function of initial velocity for our sample. Different classes of meteoroids are shown using different symbols (AC, HT, SA, and JF following the classification of Borovicka et al. 2005). The solid line and two dashed lines mark the mean beginning of average strength meteoroids and their limits. The equation of the empirical mean line is $h_{\mathrm{B}}=58 \times v^{0.156}$ (see Borovicka et al. 2005).

objects within our uncertainty. A few of the densest objects have significantly higher modelled bulk densities. As Iron is the most cosmochemically abundant element with a bulk density in this range, we presume this is a major component, though the mineralogy is quite unconstrained. These so called "iron" meteoroids have density values most closely matching stonyiron meteorites, which have grain densities around $4820 \mathrm{~kg} \mathrm{~m}^{-3}$, and porosities of $6 \%$ (Consolmagno \& Britt 1998, Table 1; Britt \& Consolmagno 2003, Table 5). It is possible that these high density $\left(\rho>4000 \mathrm{~kg} \mathrm{~m}^{-3}\right)$ meteoroids may be related to IDPs with sulfide inclusions, described by Love et al. (1994). From our measurements, these make up a large fraction of the meteoroid population at mass $(\mathrm{m}) \sim 10^{-6} \mathrm{~kg}$, possibly even higher than at IDP masses $\left(10^{-8} \mathrm{~kg}\right.$ ) (see Fig. 1 of Love et al. 1994).

Our A group contains mostly material with chondrite-like bulk densities, which for bulk meteoroid samples has a grain density of typically $3640 \mathrm{~kg} \mathrm{~m}^{-3}$, and average porosity $\sim 10 \%$ (Britt \& Consolmagno 2003). These meteoroids mappear to most closely resemble chondritic - filled (CF) IDPs (Bradley et al. 2003). The density of HTC/NIC - related particles we find to be $(350-1500) \mathrm{kg} \mathrm{m}^{-3}$. This is close to that of many IDPs which have an average density of $600 \mathrm{~kg} \mathrm{~m}^{-3}$, indicating appreciable porosity (Love et al. 1994). The cometary meteoroid bulk density is consistent with the bulk density inferred for the several cometary nuclei (cf. Asphaug \& Benz 1996; Davidson \& Gutierrez 2004, 2005) suggesting microporosity could dominate cometary nucleus structure for HTC/NICs. However, we caution that most of these measurements are for Jupiter-family comets.

The aei plot of our samples (Fig. 21) compared to Figs. 4 and 6 of Bottke et al. (2002) shows a concentration of meteoroids

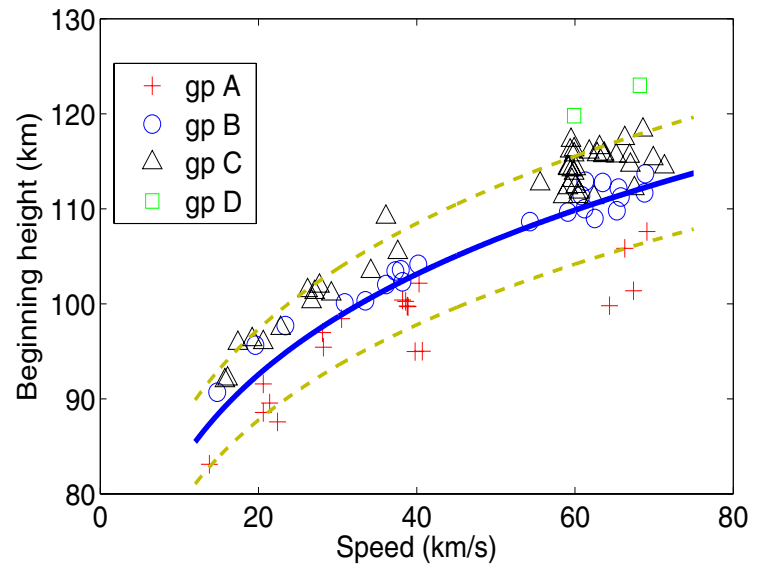

Fig. 20. Meteor beginning height as a function of initial velocity. We have plotted Ceplecha's classes of meteoroids in our sample (A-group, B-group, C-group, and D-group). The solid line and two dashed lines mark the mean beginning of average strength meteoroids and their limits, with the empirical mean line being $h_{\mathrm{B}}=58 \times v^{0.156}$ (see Borovicka et al. 2005).

originating from the $v 6$ secular resonance, and the 3:1 mean motion resonance. This suggests that most of these objects are asteroidal. Their density is uniformly high, as expected of asteroidal material.

We have also examined the relationship between our estimated bulk densities and meteoroid orbits as a function of the various parameters which are fit in our model. This included examining trends in the heat of ablation, boiling point, temperature at which the grains are relased, specific heat, average molar mass and thermal conductivity of the meteoroids as of function of bulk density and (separately) orbit-type. We found that most parameters showed no strong correlation, except thermal conductivity and bulk density.

When plotting thermal conductivity versus bulk density of our sample (Fig. 22) (we took the average of thermal conductivity in density bins of $1000 \mathrm{~kg} \mathrm{~m}^{-3}$ sizes), we find that thermal conductivity has a correlation with bulk density, as expected. All our low density meteoroids have low thermal conductivity suggesting their porous property, consistent with the thermal conductivity value assumed by Vokrouhlicky \& Farinella (2000) for porous and fragmented rocks in their work on efficient delivery of meteorites to the Earth.

\subsection{Meteor showers}

Our dataset has two noticeable shower populations: one around geocentric right ascension $\alpha_{\mathrm{g}} \sim 50^{\circ}$ and geocentric declination $\delta_{\mathrm{g}} \sim 60^{\circ}$, another around $\alpha_{\mathrm{g}} \sim 350^{\circ}$ and $\delta_{\mathrm{g}} \sim 2^{\circ}$. We used 

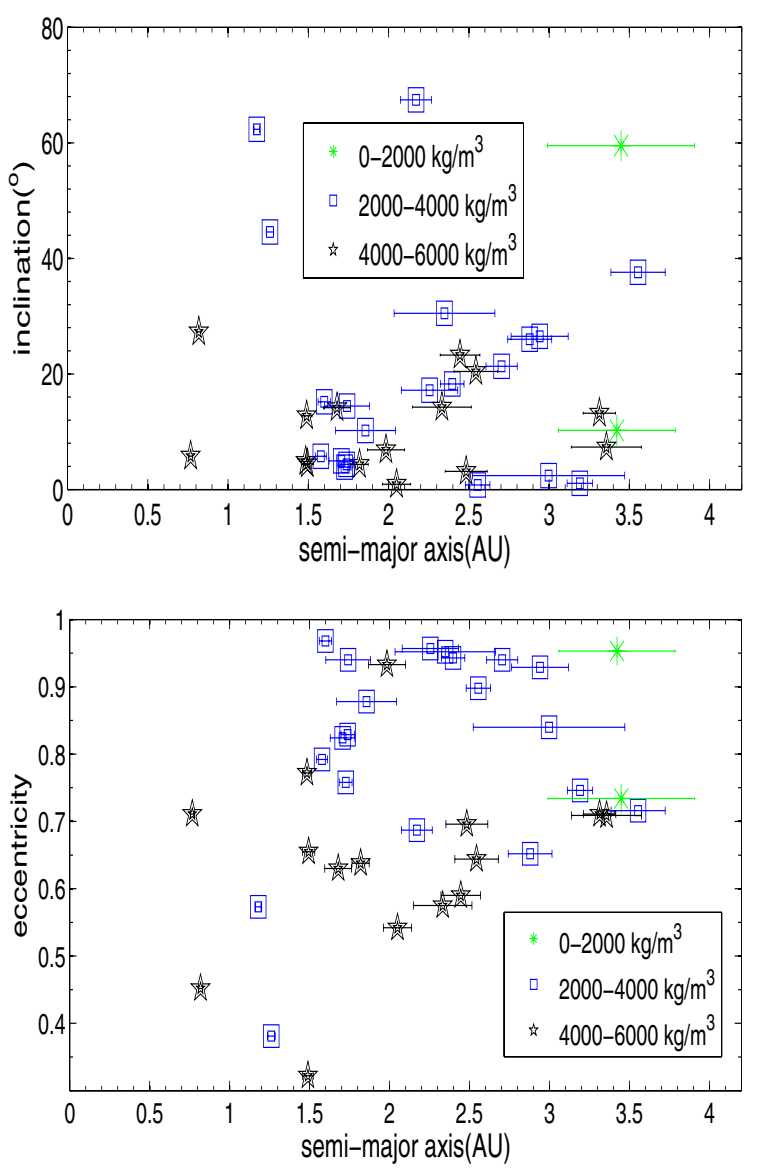

Fig. 21. Plot of aei of our sample for meteoroids with semi-major axis $\mathrm{a} \leq 4.2 \mathrm{AU}$, and inclination $\leq 80^{\circ}$.

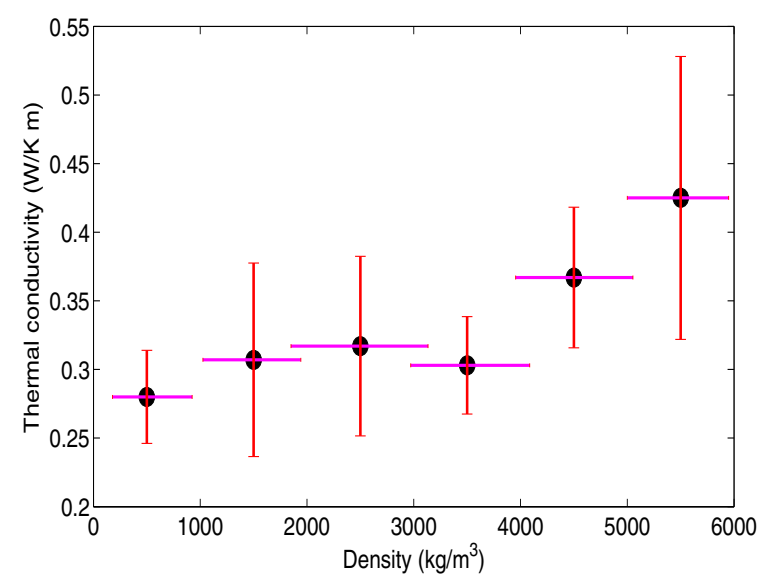

Fig. 22. Plot of Thermal conductivity versus bulk density of our sample. The data are binned in approximately $1000 \mathrm{~kg} \mathrm{~m}^{-3}$ steps (indicated by the extent of the horizontal errors bar and the vertical error bars are the standard error of the mean in that bin).

the catalog of meteor showers published by Brown et al. (2010) to identify the two concentrations of radiants: 10 meteoroids belong to the Perseids $\left(\alpha_{\mathrm{g}}=52.17^{\circ}, \delta_{\mathrm{g}}=58.07^{\circ}, v_{\mathrm{g}}=\right.$ $\left.61.9 \mathrm{~km} \mathrm{~s}^{-1}\right)$, and another 4 meteoroids to the Northen Iota Aquarids $\left(\alpha_{\mathrm{g}}=352.25^{\circ}, \delta_{\mathrm{g}}=1.84^{\circ}, v_{\mathrm{g}}=28.7 \mathrm{~km} \mathrm{~s}^{-1}\right)$.

We find the average bulk density of Perseids in our sample to be $620 \pm 200 \mathrm{~kg} \mathrm{~m}^{-3}$. This average bulk density is close to the value of $600 \mathrm{~kg} \mathrm{~m}^{-3}$ found by Bellot Rubio et al. (2002) but somewhat smaller than the $1300 \mathrm{~kg} \mathrm{~m}^{-3}$ value reported by Babadzhanov (2002; see also Borovicka 2005, Table 3). The 4 Northen Iota Aquarids found in our sample have an average bulk density of $\sim 3200 \pm 400 \mathrm{~kg} \mathrm{~m}^{-3}$. Their $K_{\mathrm{B}}$ values put them in the A-group. Their Tisserand parameters are below 3, except for one (20090825-070044) whose $T_{\mathrm{j}}=4.184$. From this small sample it appears that the Northen Iota Aquariids have closer affinities to chondritic bodies than low density cometary objects. Their high density may reflect a small $q$ in the past, and perhaps also a JF /(Encke) origin.

\section{Conclusion}

Our study combined very precise meteor observations with a complete model of meteor ablation to estimate the bulk density of very small (mean mass $\sim 10^{-6} \mathrm{~kg}$ ) optical LLLTV meteoroids. Of 107 meteors captured with three different TV systems, $78 \%$ showed noticeable deceleration, an important constraint for the ablation model. While some meteors (9 in total) saturated our Gen III cameras, a technique for saturation correction was applied in order to get an accurate photometric mass; without this correction, there would be significant error in meassured photometric mass and bulk density.

We were able to compute the bulk density of 92 meteoroids using a model of meteor ablation based on the work of Campbell-Brown \& Koschny (2004), where we required that the complete observed lightcurve and deceleration be fitted simultaneously to the modeled lightcurve and deceleration. We found that our values of bulk density covered the entire range of possible meteoroid bulk density values: from very porous cometary bodies (low density) to very high values, consistent with asteroidal bodies made largely of iron in some form.

We were able to identify in our sample the 5 different classes used by Borovicka et al. (2005): (AC): asteroidal and chondritic orbtis, (SA): Sun-approaching orbits, (JF): Jupiter family orbits, and (HT): Halley type orbits. Meteoroids with orbits belonging to the Ecliptic shower class were all members of the Northen Iota Aquariid shower. The average density of meteoroids whose orbits belong to the (AC) class was found to be $4200 \mathrm{~kg} \mathrm{~m}^{-3}$, suggesting that they are asteroidal bodies with substantial iron content.

Meteoroids with Jupiter family orbits (JF) have an average density of $3100 \mathrm{~kg} \mathrm{~m}^{-3}$. This is also the average bulk density for the 4 meteoroids with orbits belonging to the Ecliptic shower orbits (ES), consistent with the notion that the NIA derive from 2P/Encke. Both categories (JF and ES) are physically chondriticlike. The high bulk density of JFC-type meteoroids is an unexpected result. Current models suggest JF meteoroids have undergone more thermal processing than HTC or NIC-related meteoroids, the high bulk densities we find for JFCs are most consistent with chondritic-like meteorite properties or porous bodies with highly refractory inclusions. The latter interpretation is supported by observations of high bulk density IDPs (Love et al. 1994), some of which approach $7000 \mathrm{~kg} \mathrm{~m}^{-3}$, and the fact that our observed lightcurves cannot be reproduced by single body ablation of a solid chondritic stone. The existence of exclsuively high density meteoroids within this class is perhaps also related to longer collisional lifetimes of such particles. Whether such high bulk densities for small meteoroids from the JFC population as a whole are consistent with the early results from the stardust analysis of $81 \mathrm{P} /$ Wild 2 remains an open question (cf. Ishii et al. 2008), but the clear requirement for a 
substantial refractory component to match our high bulk densities supports the notion that many JFCs have incorporated substantial amounts of material formed in the inner Solar System. A possible interpretation of this result is that the process of radial transport of refractory material outward at the time of solar system formation was a common process for materials up to tens - hundreds of microns in size, supporting models of nebular formation which suggest radial transport was an integral part of protoplanetary disk dynamics (Ciesla 2007). Alternatively, the JFCs as a population formed much closer to the sun than previously thought.

Meteoroids of the HT (Halley type) class have a minimum bulk density of $360_{-100}^{+400} \mathrm{~kg} \mathrm{~m}^{-3}$ and a maximum value of $1510_{-900}^{+400} \mathrm{~kg} \mathrm{~m}^{-3}$. These bulk densities are consistent with that found for CP and cluster IDPs (Bradley 2003). They are also consistent with our notion of cometary bodies. SA (Sunapproaching)-type meteoroids show a wide spread in their densities, ranging from $1000 \mathrm{~kg} \mathrm{~m}^{-3}$ to $4000 \mathrm{~kg} \mathrm{~m}^{-3}$.

Our lack of significant numbers of meteoroids with densities of $\sim 2000-2500 \mathrm{~kg} \mathrm{~m}^{-3}$, consistent with carbonaceous chondrites, is also puzzling. While it is possible number statistics are partially responsible, this seems unlikely for a dataset approaching 92 meteoroid density measurements. On the surface, it suggests few truly carbonaceous-type materials in pure form at our sizes, in contradiction to earlier work by Ceplecha (1988), who suggests $\sim 1 / 4$ of the total meteoroid population at these masses had density $\sim 2000 \mathrm{~kg} \mathrm{~m}^{-3}$, but this was an inferred theoretical value, rather than directly measured.

We were also able to identify in our sample the 2 principal groups according to Ceplecha's classification (Ceplecha 1988): A-group, C-group, with hints of the D-group. We find no evidence for a separate peak belonging to Ceplecha's (1967) B-class, which he associated with carbonaceous chondrites. The average bulk density was found to be $4700 \mathrm{~kg} \mathrm{~m}^{-3}$ for the A-group (asteroidal meteoroids), $1000 \mathrm{~kg} \mathrm{~m}^{-3}$ for C-group, and $600 \mathrm{~kg} \mathrm{~m}^{-3}$ for D-group. These values are close to those adopted by Ceplecha $(1966,1968)$, based on photographic Super-Schmidt meteors. They are quite different from the average bulk density values of Ceplecha (1988). Two possible reasons for these differences are that we use different assumptions and a different model of ablation; as well the luminous efficiency we use is different.

We detected two different meteor showers in our sample: Perseids (10 meteoroids, $\sim 11 \%$ of our sample) and Northern Iota Aquariids (4 meteoroids). The average bulk density of Perseids was $620 \mathrm{~kg} \mathrm{~m}^{-3}$, close to Bellot Rubio et al.'s bulk density value. It is consistent with the HTC origin of Perseids, as expected.

Acknowledgements. J.B.K. thanks the Vatican Observatory for funding support and Guy Consolmagno for help in manuscript preparation. J.B.K. thanks R. J. Weryk for helping gather the observations and providing the code used for the data reduction. The authors thank Jiri Borovicka and Zdenek Ceplecha for use of their software. The authors acknowledge funding support and helpful discussions with the NASA Meteoroid Environment Office. P.G.B., M.D.C. thank the Natural Sciences and Engineering Research Council for funding support for this work. Helpful discussions with W. F. Bottke and R. Hawkes are also gratefully acknowledged.

\section{References}

Asphaug, E., \& Benz, W. 1996, Icarus, 121, 225

Babadzhanov, P. B. 2002, A\&A, 384, 317

Bellot Rubio, L. R., Martinez Gonzalez, M. J., Ruiz Herrera, L., et al. 2002, A\&A, 389, 680

Bevington, P. R., \& Robinson, D. K. 2003, Data Reduction and Error Analysis for the Physical Science (New York: McGraw-Hill)

Borovicka, J. 1990, Bull. Astr. Inst. Czechosl., 41, 391

Borovicka, J. 2005, Proceedings of the International Astronomy Union (Cambridge Univ. Press), 249

Borovicka, J., Koten, P., Spurny, P., et al. 2005, Icarus, 174, 15

Borovicka, J., Spurny, P., \& Koten, P. 2007, A\&A, 473, 661

Bottke, W. F., Morbidelli, A., Jedicke, R., et al. 2002, Icarus, 156, 399

Bradley, J. P. 2003, TrGeo, 1, 689

Britt, D. T., \& Consolmagno, G. J. 2003, M\&PS, 38, 1161

Bronshten, V. A. 1983, The Physics of Meteoritic Phenomena (Dordrecht: Reidel)

Brown, P., Weryk, R. J., Wong, D. K., et al. 2008, Icarus, 195, 317

Brown, P., Wong, D. K., Weryk, R. J., et al. 2010, Icarus, 207, 66

Brownlee, D., Tsou, P., Aleon, J., et al. 2006, Science, 314, 1711

Campbell-Brown, M. D., \& Koschny, D. 2004, A\&A, 418, 751

Ceplecha, Z. 1958, BAICz, 9, 154

Ceplecha, Z. 1966, BAICz, 17, 96

Ceplecha, Z. 1967, SCoA, 11, 35

Ceplecha, Z. 1968, SAO Special Report, 279, 1

Ceplecha, Z. 1987, BAICz, 38, 4, 222

Ceplecha, Z. 1988, BAICz, 39, 4, 221

Ceplecha, Z., Borovicka, J., Elford, W. G., et al. 1998, Space Sci. Rev., 84, 327

Ceplecha, Z., Borovicka, J., \& Spurny, P. 2000, A\&A, 357, 1115

Christou, A. A. 2010, MNRAS, 402, 2759

Ciesla, F. J. 2007, Science, 318, 613

Consolmagno, G. J., \& Britt, D. T. 1998, M\&PS, 33, 1231

Davidson, B. J. R., \& Gutierrez, P. J. 2004, Icarus, 168, 392

Davidson, B. J. R., \& Gutierrez, P. J. 2005, Icarus, 176, 453

Fernandez, J. A. 1980, Icarus, 42, 406

Fernandez, Y. R., Jewitt, D. C., \& Sheppard, S. S. 2001, ApJ, 553, 197

Fisher, A. A., Hawkes, R. L., Murray, I. S., et al. 2000, Planet. Space Sci., 48, 911

Grun, E., et al. 2002, The Observatory, 122, 128

Hawkes, R. L. 2002, Meteors in the Earth's atmosphere, ed. E. Murad, \& I. P. Williams, 97

Hawkes, R. L., \& Jones, J. 1975, MNRAS, 173, 339

Hawkes, R. L., Bussey, J. E., Macphee, S. L., et al. 2001, Proceedings of the Meteoroids 2001 Conference, 281

Hedin, A. 1991, J. Geophys. Res., 96, 1154

Hill, K. A., Rogers, L. A., \& Hawkes, R. L. 2005, A\&A, 444, 615

Horz, F., Bastien, R., Borg, J., et al. 2006, Science, 314, 1716

Ishii, H. A., Bradley, J. P., Dai, Z. R., et al. 2008, Science, 319, 447

Jacchia, L. G. 1955, ApJ, 121, 521

Jones, J., \& Kaiser, T. R. 1966, MNRAS, 133, 411

Kikwaya, J. B., Campbell-Brown, M., Brown, P. G., et al. 2009, A\&A, 497, 851

Kikwaya, J. B., Weryk, R. J., Campbell-Brown, M., et al. 2010, MNRAS, 404, 387

Koten, P., Borovička, J., Spurny, P., et al. 2004, A\&A, 428, 683

Koten, P., Spurny, P., Borovicka, J., et al. 2006, M\&PS, 41, 1271

Levin, B. J. 1956, BAICz, 7, 58

Levison, H. F. 1996, in Completing the Inventory of the Solar System, ed. T. W. Rettig, \& J. M. Hahn, ASP Conf. Proc., 107, 173

Levison, H. F., \& Duncan, M. J. 1994, Icarus, 108, 18

Love, S. G., Joswiak, D. J., \& Brownlee, D. E. 1994, Icarus, 111, 227

Morbidelli, A. 2008, Saas-Fee Advanced Course 35 ed. D. Jewitt, A. Mordidelli, \& H. Rauer, 79

Murray, I. S., Beech, M., Taylor, M. J., et al. 2000, EM\&P, 82/83, 351

Myers, J. R., Sande, C. B., Miller, A. C., et al. 2001, Sky 2000 Catalog v4, Goddard Space Flight Center, Ycat 5109

Popova, O. P. 2004, EM\&P, 95, 303

Sarma, T., \& Jones, J. 1985, BAICz, 36, 9

Vokrouhlicky, D., \& Farinella, P. 2000, Nature, 407, 606

Weissman, P. R., Bottke, Jr, W. F., \& Levison, H. F. 2002, Asteroids III, University of Arizona, Tucson, 669 